\title{
HEAVY FLAVOUR PHYSICS FROM TOP TO BOTTOM
}

\author{
Manfred Paulini \\ Lawrence Berkeley National Laboratory \\ MS 50B-5239, Berkeley, California 94720 \\ (Representing the CDF and DØ Collaboration)
}

\begin{abstract}
We review the status of heavy flavour physics at the Fermilab Tevatron collider by summarizing recent top quark and $B$ physics results from CDF and DØ. In particular we discuss the measurement of the top quark mass and top production cross section as well as $B$ meson lifetimes and time dependent $B \bar{B}$ mixing results. An outlook of perspectives for top and $B$ physics in Run II starting in 1999 is also given.
\end{abstract}




\section{Introduction}

In this article we review recent heavy flavour physics results from the Tevatron $p \bar{p}$ collider at Fermilab, where heavy flavour refers to the top quark as well as the bottom quark. After a brief historical overview we summarize the status of top quark physics at $\mathrm{CDF}$ and $\mathrm{D} \varnothing$ in Sec. 2. In particular we discuss the measurement of the top production cross section and the top quark mass. Section 3 is devoted to recent $B$ physics results at a hadron collider, where we concentrate on $B$ hadron lifetimes and latest time dependent $B \bar{B}$ mixing results. A brief look to the future, summarizing the prospects of top quark physics as well as $B$ physics and $C P$ violation in Run II starting in 1999, is given in Sec. 4 . We conclude with Section 5 .

\section{$1.1 \quad$ Historical Overview}

In 1977 the bottom quark was discovered as a resonance in the dimuon mass spectrum in $400 \mathrm{GeV}$ proton-nucleus collisions at Fermilab.11 Soon after the discovery the so-called $\Upsilon$ resonances at a mass of about $9.5 \mathrm{GeV} / c^{2}$ were confirmed in $e^{+} e^{-}$collisions at the DORIS storage ring at DESY $⿴$. From the narrow resonance observed at $(9.46 \pm 0.01) \mathrm{GeV} / c^{2}$ the PLUTO and DASP detectors were able to determine the electronic width $\Gamma_{e e}$, which implied a charge assignment of $-1 / 3$ for the $b$ quark. It took a few years until decays of $B$ mesons, bound states of a $b$ quark and a light quark, were observed in 1983 by the CLEO collaboration. ${ }^{3}$ The weak isospin of the $b$ quark was first extracted from the forward-

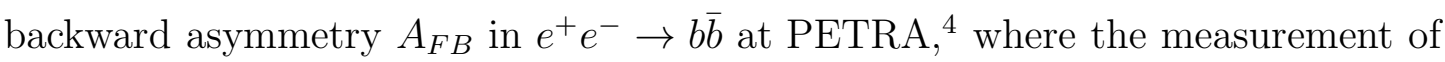
$A_{F B}=(-22.8 \pm 6.0 \pm 2.5) \%$ at $\sqrt{s}=34.6 \mathrm{GeV}$ was found to be consistent with the Standard Model prediction of $A_{F B}=-25 \%$ assuming $I_{3}=-1 / 2$ for the weak isospin of the $b$ quark. This indicated that the bottom quark has a partner and it can be counted as the first evidence for the existence of the top quark. Finally, concluding this brief historical overview with a link between top and $B$ physics, $B^{0} \bar{B}^{0}$ mixing was first observed by the ARGUS collaboration in 1987. surement of a large mixing parameter $x_{d}$ was the first indication of a large top quark mass.

Why are we discussing the history of $b$ quark physics? A certain pattern can be observed after the discovery of a new quark. This pattern appears to repeat itself after the discovery of the top quark. It looks like right after the discovery of the 
$b$ quark an attempt was made to confirm the discovery in a different environment like $e^{+} e^{-}$collisions. Then the fundamental quantities of the newly discovered quark, like its charge and isospin, were determined. After sufficient statistics is accumulated rare phenomena like $B \bar{B}$ oscillations were searched for. Nowadays, $B$ physics is fully explored at $e^{+} e^{-}$colliders and rare phenomena like $b \rightarrow u$ transitions or $b \rightarrow s \gamma$ penguin decays are also studied 6 This raises the question of why $B$ physics is studied at a $p \bar{p}$ hadron collider which is a much more difficult environment to study low $p_{\mathrm{t}}$ physics. We shall discuss this issue further on, in Section 3 .

\subsection{Early Searches for the Top Quark}

The experimental search for the top quark begun soon after the discovery of the $b$ quark. Between 1979 and 1984 measurements of $R$, the ratio of the cross sections $\sigma\left(e^{+} e^{-} \rightarrow\right.$ hadrons $)$ to $\sigma\left(e^{+} e^{-} \rightarrow \mu^{+} \mu^{-}\right)$, were performed at the PETRA $e^{+} e^{-}$ storage ring up to a centre-of-mass energy of $46.8 \mathrm{GeV}$. The value of $R$ was found to be consistent with Standard Model predictions without a top quark contribution setting a lower bound on the top quark mass of $>23.3 \mathrm{GeV} / c^{2}$. Later searches at $e^{+} e^{-}$colliders were also negative and limits on $m_{\text {top }}$ of half of the centre-of-mass energy were set. These limits from TRISTAN as well as SLd 9 and LEP 10 are listed in Table 1 .

With the advantage of higher mass regions being accessible, the search for the top quark was soon dominated by $p \bar{p}$ colliders, first at the SPS collider $(\sqrt{s}=$ $630 \mathrm{GeV})$ at CERN and then at the Tevatron $(\sqrt{s}=1.8 \mathrm{TeV})$ at Fermilab. Initial results reported in 1984 by the UA1 collaboration 11 at the SPS seemed to be consistent with the production of a top quark of mass $(40 \pm 10) \mathrm{GeV} / c^{2}$ in $p \bar{p} \rightarrow W \rightarrow t \bar{b}$. The results were based on the observation of 12 isolated lepton plus 2-jet events with an expected background of approximately 3.5 events in $200 \mathrm{nb}^{-1}$. However, these first results were not supported by a subsequent UA1 analysis 12 with a higher statistics data sample setting a lower limit on $m_{\text {top }}$ of $>52 \mathrm{GeV} / c^{2}$. More sensitive searches were performed later on by UA2 13 and

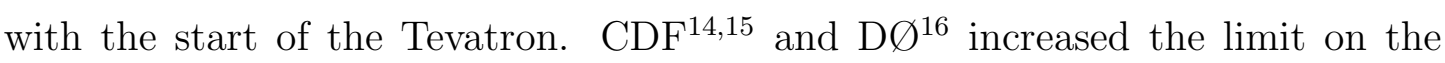
mass of the top quark to finally $>131 \mathrm{GeV} / c^{2}$ in 1994 (see Table 1).

However, in April 1994 the CDF collaboration presented evidence for top

quark production 17.18 with the observation of 12 events consistent with either 


\begin{tabular}{|c|cl|c|l|}
\hline Year & \multicolumn{2}{|c|}{ Location } & Mass limit $(95 \% \mathrm{CL})$ & Ref. \\
\hline \hline 1979 & $e^{+} e^{-}:$ & PETRA & $>23.3 \mathrm{GeV} / c^{2}$ & G \\
1987 & $e^{+} e^{-}:$ & TRISTAN & $>30.2 \mathrm{GeV} / c^{2}$ & B \\
1989 & $e^{+} e^{-}:$ & SLC \& LEP & $>45.8 \mathrm{GeV} / c^{2}$ & 9.10 \\
\hline 1984 & $p \bar{p}:$ & $\mathrm{UA} 1$ & $(40 \pm 10) \mathrm{GeV} / c^{2}$ & [1 \\
1988 & $p \bar{p}:$ & $\mathrm{UA} 1$ & $>52 \mathrm{GeV} / c^{2}$ & 12 \\
1990 & $p \bar{p}:$ & $\mathrm{UA} 2$ & $>69 \mathrm{GeV} / c^{2}$ & 13 \\
1990 & $p \bar{p}:$ & $\mathrm{CDF}$ & $>77 \mathrm{GeV} / c^{2}$ & 口4 \\
1992 & $p \bar{p}:$ & $\mathrm{CDF}$ & $>91 \mathrm{GeV} / c^{2}$ & 15 \\
1994 & $p \bar{p}:$ & $\mathrm{D} \varnothing$ & $>131 \mathrm{GeV} / c^{2}$ & 16 \\
\hline
\end{tabular}

Table 1: Historical overview of searches for the top quark and limits on the top quark mass.

two $W$ bosons, or a $W$ boson and at least one $b$ jet. The probability that the measured yield was consistent with the expected background was $0.26 \%$ corresponding to a $2.8 \sigma$ effect. Finally, in February 1995 the top quark was discovered by the CDF experiment 19 and the D $\varnothing$ experiment 20 at the same time. Although top quark physics is still a relatively young field at the time of this conference, a lot of progress has been made in understanding the top quark and its characteristics.

\section{Status of Top Quark Physics at CDF and DØ}

\subsection{The Tevatron with the CDF and DØ Detectors}

At the Fermilab Tevatron, proton-antiproton collisions take place at a centreof-mass energy of $\sqrt{s}=1.8 \mathrm{TeV}$. The Tevatron Run I started delivering data in Dec. 1992 and finished in Feb. 1996. During this period a total of about $110 p \mathrm{~b}^{-1}$ and $100 \mathrm{pb}^{-1}$ of data were accumulated by the CDF and DØ experiment, respectively. All results presented in this paper refer to the full Run I statistics unless otherwise noted. The running period was devided up in a so-called Run Ia from Dec. 1992 through Aug. 1993 and Run Ib from Dec. 1993 to Feb. 1996. The collected integrated luminosities by $\mathrm{CDF}$ and $\mathrm{D} \varnothing$ were $\approx 19.3 p \mathrm{~b}^{-1}$ and $\approx 15 p \mathrm{~b}^{-1}$ for Run Ia as well as $\approx 90 \mathrm{pb}^{-1}$ and $\approx 85 \mathrm{pb}^{-1}$ for Run Ib, respectively. During that time the Tevatron operated with six bunches of protons and six bunches of antiprotons crossing every $3.5 \mu \mathrm{s}$ at the experiments interaction regions. During 
(a)

\section{CDF Detector}

(b)

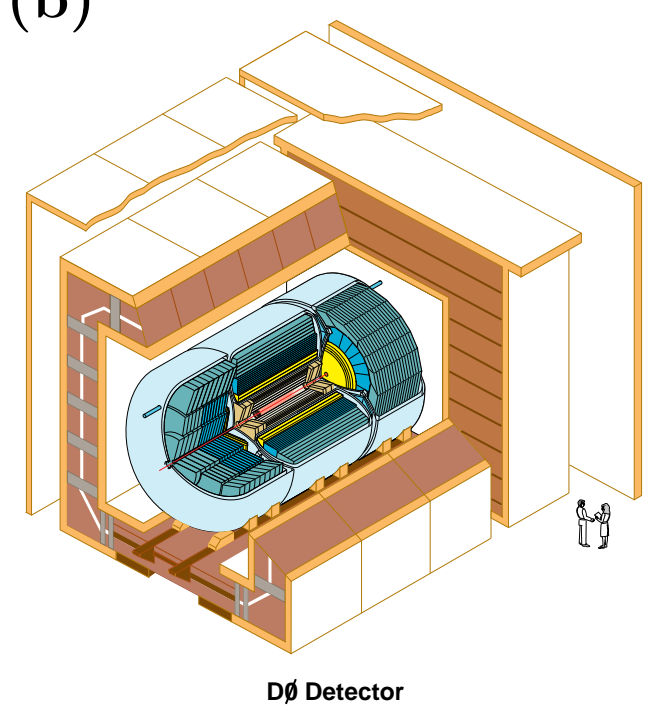

Figure 1: Schematic view of (a) the CDF detector and (b) the DØ experiment.

Run Ib the highest instantaneous luminosities, which were reached, were around $2.5 \cdot 10^{31} \mathrm{~cm}^{-2} \mathrm{~s}^{-1}$. At this luminosity on average two interactions accured per beam crossing.

\subsubsection{The CDF Detector}

The Collider Detector at Fermilab (CDF) is a general purpose detector to efficiently identify leptons and hadronic jets, as well as charged particles in $p \bar{p}$ collisions. 2 A schematic view of the CDF detector is shown in Figure 17a). Three devices inside the $1.4 \mathrm{~T}$ solenoid are used for the tracking of charged particles: the silicon vertex detector (SVX), a set of vertex time projection chambers (VTX), and the central tracking chamber (CTC).

The SVX consists of four layers of silicon microstrip detectors located at radii between $3.0 \mathrm{~cm}$ and $7.9 \mathrm{~cm}$ from the interaction point and provides spatial measurements in the $r-\varphi$ plane with a resolution of $13 \mu \mathrm{m}$, giving a track impact parameter resolution of about $\left(13+40 / p_{\mathrm{t}}\right) \mu \mathrm{m}$, 2 where $p_{\mathrm{t}}$ is the transverse momentum of the track in $\mathrm{GeV} / c$. Throughout this article $\varphi$ is the azimuthal angle, $\theta$ is the polar angle measured from the proton direction, and $r$ is the radius from the beam axis ( $z$-axis). The geometric acceptance of the SVX is $\sim 60 \%$ as it extends to only $\pm 25 \mathrm{~cm}$ from the nominal interaction point whereas the Tevatron beam has an RMS width of $\sim 30 \mathrm{~cm}$ along the beam direction. The transverse 
profile of the beam is circular and has an RMS of $\sim 25-35 \mu \mathrm{m}$.

The VTX reconstructs intermediate tracks in the $r-z$ plane and is used to determine the primary interaction vertex. Surrounding the SVX and VTX is the CTC, a cylindrical drift chamber containing 84 layers grouped into nine alternating superlayers of axial and stereo wires. It covers the pseudorapidity interval $|\eta|<1.1$, where $\eta=-\ln [\tan (\theta / 2)]$. The $p_{\mathrm{t}}$ resolution of the CTC combined with the SVX is $\sigma\left(p_{\mathrm{t}}\right) / p_{\mathrm{t}}=\left((0.0066)^{2}+\left(0.0009 p_{\mathrm{t}}\right)^{2}\right)^{1 / 2}$, with $p_{\mathrm{t}}$ measured in $\mathrm{GeV} / c$.

Outside the solenoid are electromagnetic (CEM) and hadronic (CHA) calorimeters $(|\eta|<1.1)$ that employ a projective tower geometry with a segmentation of $\Delta \eta \times \Delta \varphi \sim 0.1 \times 15^{\circ}$. The sampling medium is composed of scintillators layered with lead and steel absorbers. A layer of proportional wire chambers (CES) is located near shower maximum in the CEM and provides a measurement of electromagnetic shower profiles in both the $\varphi$ and $z$ directions. Plug and forward calorimeters instrument the region of $1.1<|\eta|<4.2$ and consist of gas proportional chambers as active media and lead and iron as absorber materials. The overall resolution for the CDF central calorimeter is $\sigma_{E} / E=\left(13.5 \% / \sqrt{E_{\mathrm{t}}}\right)+2 \%$ for electromagetic showers and $\sigma_{E} / E=\left(75 \% / \sqrt{E_{\mathrm{t}}}\right)+3 \%$ for hadrons.

Several muon subsystems in the central region are used. The central muon chambers (CMU) and the central muon upgrade chambers (CMP) cover $80 \%$ for $|\eta| \leq 0.6$, while the central muon extention chambers (CMX) extent the coverage up to $|\eta|<1$.1. The CMP chambers are located behind eight interaction lengths of material.

\subsubsection{The DØ Detector}

The D $\varnothing$ detector 23 consists of three primary systems: a nonmagnetic tracking device, a uranium-liquid argon calorimeter, and a muon spectrometer. A perspective view of the $\mathrm{D} \varnothing$ detector can be seen in Fig. 1 b). The tracking system consists of four detector subsystems: a 3-layer vertex drift chamber, a transition radiation detector for additional electron identification, a 4-layer central drift chamber, and two forward drift chambers. The tracking system provides charged particle tracking over the region $|\eta|<3.2$.

The hermetic, compensating, uranium-liquid argon sampling calorimeter is divided into three parts: a central calorimeter and two end calorimeters. They each consist of an electromagnetic section, a fine hadronic section, and a coarse 
hadronic section, housed in a steel cryostat. The calorimeter covers the pseudorapidity range $|\eta|<4.2$ with fine longitudinal segmentation (8 depth segments) and fine transverse segmentation $\left(\Delta \eta \times \Delta \phi=0.1 \times 6^{\circ}\right.$, and $\Delta \eta \times \Delta \phi=0.05 \times 6^{\circ}$ in the third depth segment of the electromagnetic calorimeter). The overall resolution for the $\mathrm{D} \varnothing$ calorimeter is $\sigma_{E} / E=(15 \% / \sqrt{E})+0.4 \%$ for electromagetic showers and $\sigma_{E} / E=(50 \% / \sqrt{E})$ for hadrons.

The muon system, used for the identification of muons and determination of their trajectories and momenta, consists of five separate solid-iron toroidal magnets, together with sets of proportional drift tube chambers. The muon system covers $|\eta|<3.3$. The material in the calorimeter and iron toroids combined varies between 13 and 19 interaction lengths. The achieved momentum resolution is $\sigma_{p} / p=0.2+0.003 p$ (with $p$ measured in $\mathrm{GeV} / c$ ) for the rapidity range $|\eta|<3.3$.

\subsection{Top Quark Production at the Tevatron}

In $p \bar{p}$ collisions at $\sqrt{s}=1.8 \mathrm{TeV}$, the dominant top quark production mechanism is $t \bar{t}$ pair production through $q \bar{q}$ annihilation. Gluon-gluon fusion, which will be the main production mechanism at LHC energies, contributes to about $10 \%$ at the Tevatron. The production of single top quarks through the creation of a virtual $W$ boson is estimated to be about one order of magnitude lower than the $t \bar{t}$ pair production at $\sqrt{s}=1.8 \mathrm{TeV}$.

During the Tevatron Run I about $5 \cdot 10^{12} p \bar{p}$ collisions occured within the CDF and $\mathrm{D} \varnothing$ detectors but only about $500 t \bar{t}$ pairs have been produced. The top quark production cross section is about ten orders of magnitudes lower than the total inelatic cross section at the Tevatron. Comparing $\sigma_{t \bar{t}}$ to other physics processes like $W$ boson production shows that $\sigma_{t \bar{t}}$ is still three orders of magnitude lower than the $W$ cross section. This means the challenge in discovering and studying top quarks is to separate them from backgrounds in hadron collisions.

\subsection{Top Quark Decay Signature}

Within the Standard Model, each of the pair produced top quarks decays almost exclusively into a $W$ boson and a $b$ quark as shown in Fig. 2. The $W$ boson decays into either a lepton-neutrino or quark-antiquark pair. The top decay signature depends primarily on the decay of the $W$ boson. Events are classified by the 


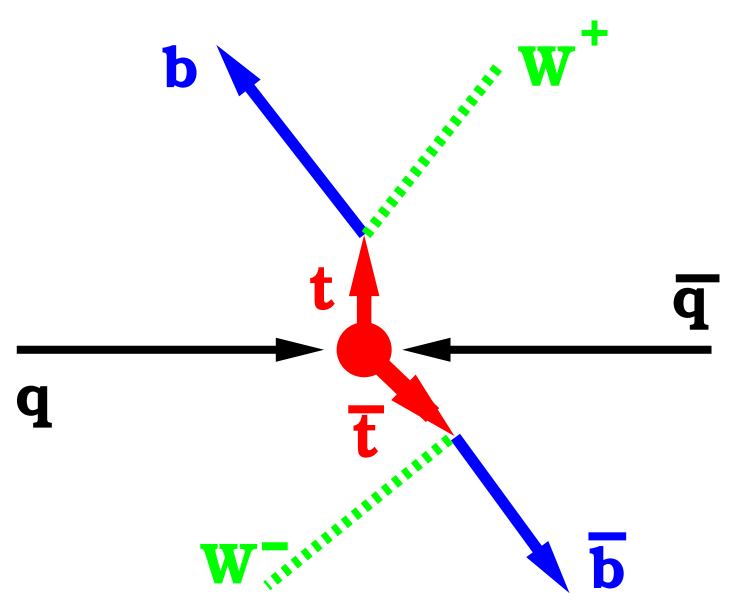

Figure 2: Top decay signature within the Standard Model.

number of $W$ 's that decay leptonically. In this context lepton refers to an electron or a muon.

If both $W$ bosons decay leptonically into $W \rightarrow \ell \nu$, we call it the 'dilepton channel', where the final state consists of $\ell^{-} \bar{\nu} \ell^{+} \nu b \bar{b}$ as can be seen in Figure 3a). This means there will be two leptons and two jets originating from $b$ quarks in the event. Due to both $W$ 's decaying semileptonically, this top decay mode has a small branching fraction of about $5 \%$.

If one of the $W$ bosons decays leptonically into $W \rightarrow \ell \nu$ and the other into $W \rightarrow q \bar{q}^{\prime}$, we call it the 'lepton plus jets channel', where the final state consists of $\ell \nu q \bar{q}^{\prime} b \bar{b}$ as shown in Figure $3 \mathrm{~b}$ ). In this case one lepton and four jets, where two jets originate from $b$ quarks, can be seen in the event. This decay mode happens in about $30 \%$ of the time.

If both $W$ bosons decay into quark pairs as $W \rightarrow q \bar{q}^{\prime}$, we call it the 'all hadronic channel'. The final state consists of $q \bar{q}^{\prime} q \bar{q}^{\prime} b \bar{b}$ (see Fig. (4). In this case we can find six jets in the event, where two jets originate from $b$ quarks. This top decay mode occurs at a large rate of about $44 \%$.

In additon there are about $21 \%$ of $t \bar{t}$ decays to final states containing $\tau$ leptons. According to the $\tau$ decay these top decays are either classified as dilepton or lepton plus jet events, if the tau decays into $e$ or $\mu$ or as all hadronic events if the tau decays hadronically. In the following we are going to use these decay channels to discuss top decays at the Tevatron. 
(a)

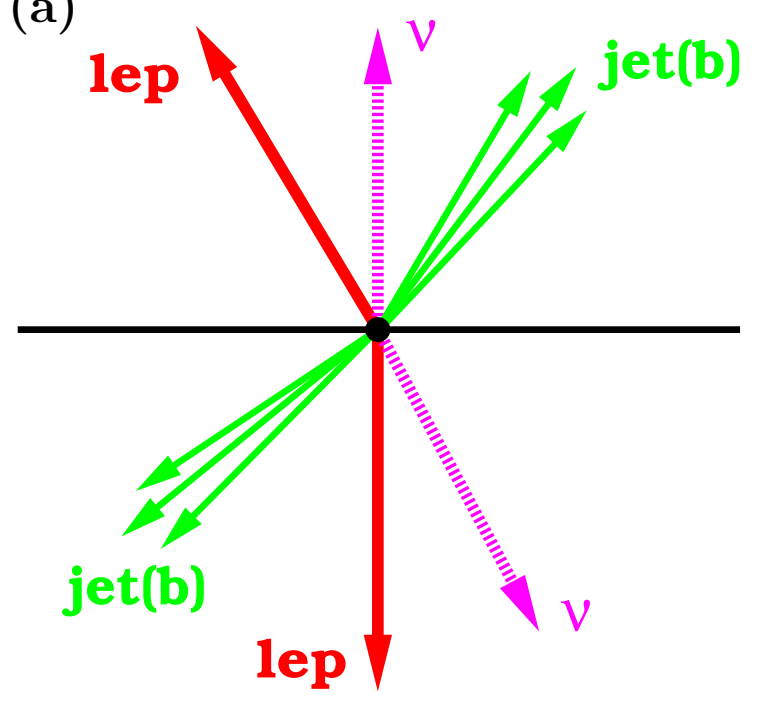

(b)

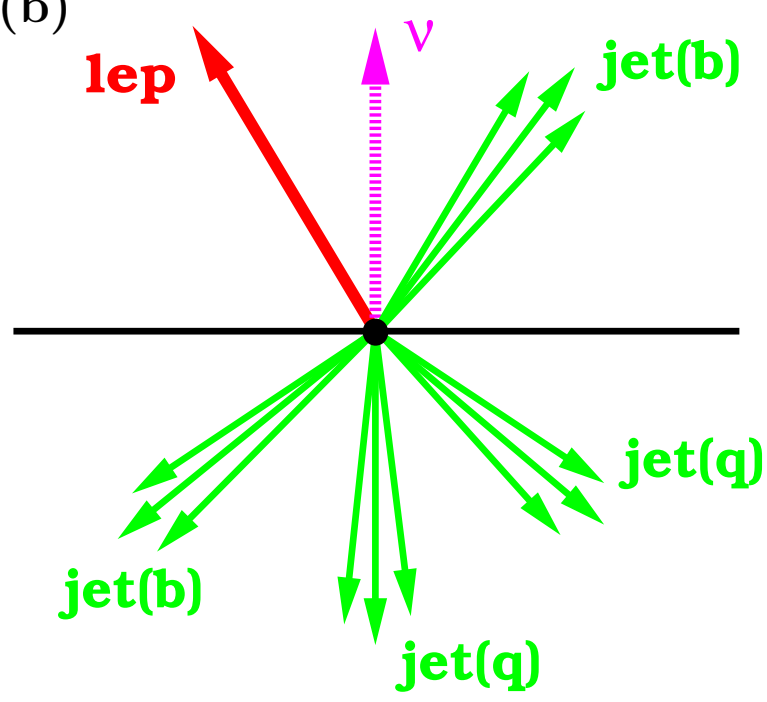

Figure 3: Top decay signature of (a) the dilepton channel and (b) the lepton plus jets channel.

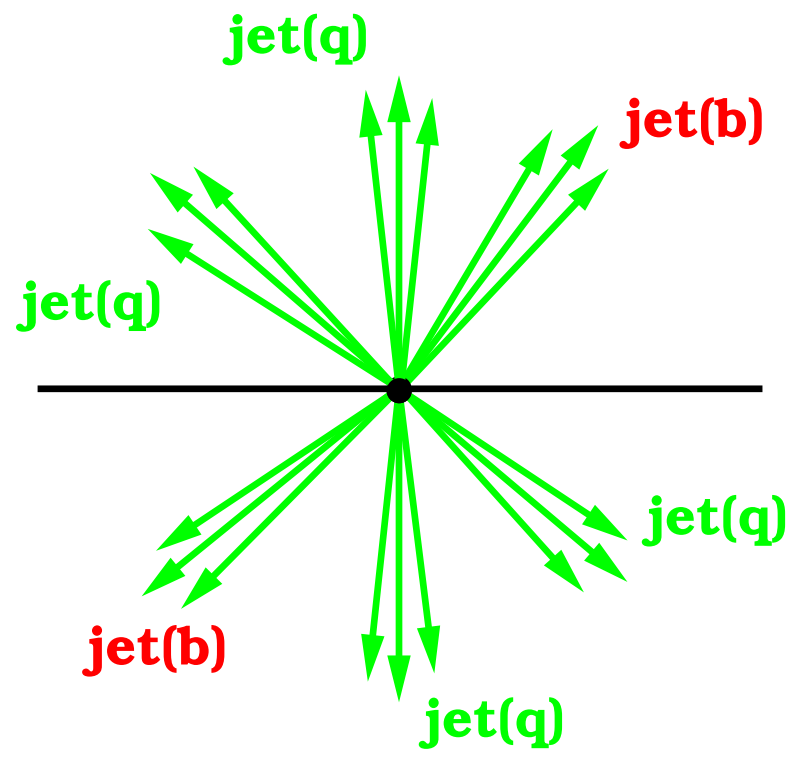

Figure 4: Top decay signature of the all hadronic channel. 


\begin{tabular}{|l||c|c|}
\hline Sample & D & CDF \\
\hline \hline$e \mu$ & 3 & 7 \\
Background & $0.4 \pm 0.1$ & $0.76 \pm 0.21$ \\
Expected Yield & $1.7 \pm 0.3$ & $2.4 \pm 0.2$ \\
\hline$e e$ or $\mu \mu$ & 2 & 2 \\
Background & $1.2 \pm 0.4$ & $1.23 \pm 0.36$ \\
Expected Yield & $1.4 \pm 0.1$ & $1.6 \pm 0.2$ \\
\hline$e$ or $\mu+\tau$ & 2 & 4 \\
Background & $1.4 \pm 0.5$ & $1.96 \pm 0.35$ \\
Expected Yield & $1.4 \pm 0.1$ & $0.7 \pm 0.1$ \\
\hline
\end{tabular}

Table 2: Event summary for the dilepton channel. The expected yield is based on determinations of the top cross section 24 for a top quark mass of $175 \mathrm{GeV} / c^{2}$.

\subsection{The Top Dilepton Channel}

The signature of the dilepton channel (see Fig. 3a) is two isolated high $p_{\mathrm{t}}$ leptons (e or $\mu$ ) and missing energy $\left(E_{\mathrm{t}}\right)$ from the two neutrinos that escape the detector unobserved. In addition, two or more jets can be found in the event. The event selection also relies on kinematic requirements. The dominant backgrounds are from $W W$ diboson production plus jet activity where both $W$ 's decay leptonically and from jet plus $Z$ production with $Z \rightarrow \tau \tau$ followed by subsequent leptonic $\tau$ decays. Events where the $e^{+} e^{-}$or $\mu^{+} \mu^{-}$invariant mass is within $75 \mathrm{GeV} / c^{2}<$ $m_{\ell \ell}<105 \mathrm{GeV} / c^{2}$ are considered as $Z \rightarrow \ell \ell$ candidates and removed in the event selection. Fake leptons and Drell Yan production of lepton pairs are further sources of background. These backgrounds are estimated from data as well as Monte Carlo predictions.

The dilepton channel has a good signal to background ratio, but low statistics. Due to the two neutrinos, this channel is not ideal for a determination of the top quark mass. The dilepton event summary for the CDF and D $\varnothing$ experiment is shown in Table 2. Both experiments find a few events on small backgrounds in the $e \mu$, ee or $\mu \mu$, and $e$ or $\mu$ plus $\tau$ mode. The expected division of the dilepton signal events is consistent with the data observed by CDF and D $\varnothing$. For example, in the case of $\mathrm{CDF} 58 \% e \mu, 27 \% \mu \mu$, and $15 \%$ ee events are expected. In Tab. 2 the expected yield from $t \bar{t}$ production is also listed. It is based on determinations of the top cross section 24 for a top quark mass of $175 \mathrm{GeV} / c^{2}$. 


\subsection{The Top Lepton plus Jets Channel}

The signature of the lepton plus jets channel (see Fig. [3b) is one isolated high $p_{\mathrm{t}}$ lepton $\left(e\right.$ or $\mu$ ), missing energy $\left(E_{\mathrm{t}}\right)$ from the neutrino and four jets where two of them are from $b$ quarks. The dominant backgrounds are from $W$ plus jet production including $W$ plus $b \bar{b}$ production. In this channel it is important to reduce the backrounds $(S: B \approx 1: 4)$, where both experiments follow different strategies.

To reduce background, CDF tags the $b$ jets in the event through a so-called 'soft lepton tag' and a 'SVX tag'. The first technique identifies $b$ jets by searching for a lepton from $b \rightarrow \ell X$ or $b \rightarrow c \rightarrow \ell X$ decays, which have branching fractions of about $10 \%$ each. Since these leptons typically have lower momenta than the leptons from the primary $W$ decay, this technique is known as 'soft lepton tag' (SLT). It looks for electrons and muons by matching tracks from the central drift chamber with electromagnetic energy clusters in the calorimeter or track segments in the muon chambers. The $p_{\mathrm{t}}$ threshold is at $2 \mathrm{GeV} / c$. The efficiency for SLT tagging a $t \bar{t}$ event is $(18 \pm 2) \%$, and the typical fake rate per jet is about $2 \%$. Details of the SLT algorithm can be found in Ref 18

The second, more powerful $b$ tagging technique exploits the finite lifetime of $b$ hadrons by searching for a secondary decay vertex with CDF's silicon vertex detector. This technique is known as the 'SVX tag'. The algorithm begins by searching for displaced vertices containing three or more tracks which satisfy a loose set of track quality requirements. If no such vertices are found in an event, two-track vertices that satisfy more stringent quality cuts are accepted. A jet is defined to be tagged if it contains a secondary vertex which is displaced from the primary vertex with a significance of greater than three. The efficiency for SVX tagging a $t \bar{t}$ event is $(41 \pm 4) \%$, while the fake rate is only $\approx 0.5 \%$. More information on the SVX tag can be found in Ref.18 19 An example of a SVX tagged event display can be found in Fig. 5. Both $b$ jets are SVX tagged and well separated from the primary interaction vertex by $2.2 \mathrm{~mm}$ and $4.5 \mathrm{~mm}$, respectively.

The $\mathrm{D} \emptyset$ experiment makes use of two different approaches to reduce the background in the lepton plus jets channel. DØ uses kinematic and topological cuts as well as $b$ tagging via soft muon tagging. The first approach exploits the fact that the large top quark mass gives rise to kinematically different events. Jets from $t \bar{t}$ decays tend to be more energetic and more central than from typical QCD back- 


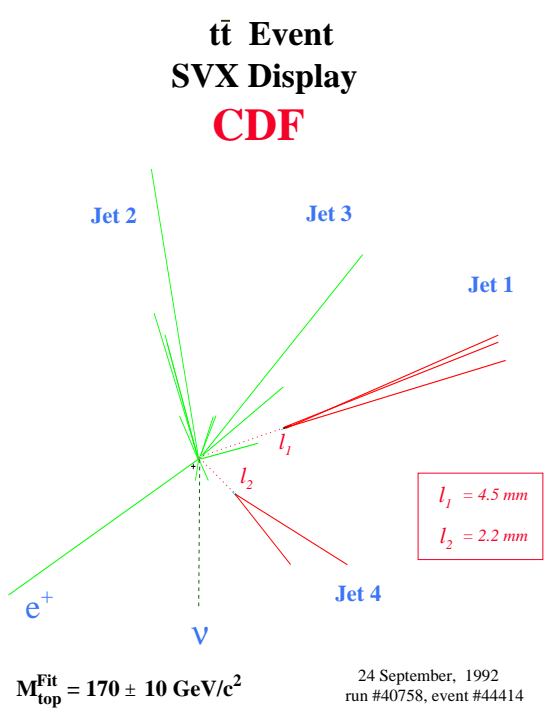

Figure 5: Example of a SVX tagged event where both $b$ jets are SVX tagged.

ground events. In addition $t \bar{t}$ events as a whole are more spherical while QCD jet production results in more planar event shapes. Top enriched data samples can therefore be selected with a set of topological and kinematic cuts like the total hadronic activity in the event, $H_{T}=\sum E_{\mathrm{t}}^{\mathrm{jet}}$, which can be combined with the aplanarity $\mathcal{A}$ of the $W$ plus jets system. A value of $\mathcal{A}=0$ indicates a planar event shape, while $\mathcal{A}=1 / 2$ reflects a spherical event shape. In addition, a third kinematic variable with discrimating power, the total leptonic transverse energy, $E_{\mathrm{t}}^{L}=E_{\mathrm{t}}^{\mathrm{lep}}+E_{\mathrm{t}}$, is also used. The second D $\varnothing$ approach uses $b$ tagging via muon tags through $b \rightarrow \mu X$ and $b \rightarrow c \rightarrow \mu X$ decays. The typical fake rate for background events is at the $\approx 2 \%$ level for tagging muons of $p_{\mathrm{t}}>4 \mathrm{GeV} / c$. For more details on both techniques see Ref.20

The lepton plus jets event summary for the CDF and D $\varnothing$ experiment is shown in Table 3. CDF finds 34 events with at least one SVX tag on a background of $(8.0 \pm 1.4)$ events, while $(19.8 \pm 4.0)$ events are expected from $t \bar{t}$ production, where a top quark mass of $175 \mathrm{GeV} / c^{2}$ has been assumed. The CDF events shape analysis is based on only $67 \mathrm{pb}^{-1}$.

\subsection{The Top All Hadronic Channel}

The signature of the all hadronic channel (see Fig. 团) is nominally six jets where two of them are from $b$ quarks, no leptons, and low $E_{t}$. Since not all jets are always 


\begin{tabular}{|l||c|c|}
\hline Sample & D & CDF \\
\hline Event Shape: & 21 & 22 \\
Observed & $9.2 \pm 2.4$ & $7.2 \pm 2.1$ \\
Background & $12.9 \pm 2.1$ & - \\
Expected Yield & 11 & 40 \\
\hline Lepton tag (SLT): & $2.5 \pm 0.4$ & $24.3 \pm 3.5$ \\
\hline Observed & $5.2 \pm 1.0$ & $9.6 \pm 1.7$ \\
Background & & \\
Expected Yield & - & 34 \\
\hline Displaced Vertex (SVX tag): & - & $8.0 \pm 1.4$ \\
\hline Observed & - & $19.8 \pm 4.0$ \\
Background & & \\
Expected Yield &
\end{tabular}

Table 3: Event summary for the lepton plus jets channel. The expected yield is based on determinations of the top cross sectionet for a top quark mass of $175 \mathrm{GeV} / c^{2}$.

observed, a jet multiplicity of at least five is required. In order to overcome the huge background from QCD multijet production, $b$ tagging alone is not sufficient and kinematic cuts are used in addition. If the backgrounds can be controlled, the all hadronic channel would be the ideal way to determine the top mass because no neutrinos are present and all objects of the top decay are measured in the detector.

After a set of kinematic cuts is applied in the CDF all hadronic analysis, at least five jets are required, where the leading jets have to pass an aplanarity cut. In addition at least one jet has to be SVX tagged. The efficiency of a SVX tag in a $t \bar{t}$ event is $(47 \pm 5) \%$ in the all hadronic mode, slightly larger than in the lepton plus jets channel due to the presence of additional charm tags from $W \rightarrow c \bar{s}$. CDF observes 192 events on a predicted background of $137 \pm 11$ events as can be seen in Table 田.

Recently, DØ also reported on a search for $t \bar{t}$ pairs in the all hadronic channel. They require at least six jets within $|\eta|<2$ and use several additional kinematic quantities. Finally, a soft muon tag has to be present in at least one of the jets. $15 \mu$ tagged events are observed for an expected background of $11 \pm 2$ events (see Table (1). 


\begin{tabular}{|l||c|c|}
\hline Sample & D & CDF \\
\hline Observed & 15 & 192 \\
Background & $11 \pm 2$ & $137.1 \pm 11.3$ \\
Expected Yield & $4.5 \pm 0.5$ & $26.6 \pm 9.1$ \\
\hline
\end{tabular}

Table 4: Event summary for the all hadronic channel. The expected yield is based on determinations of the top cross section 24 for a top quark mass of $175 \mathrm{GeV} / c^{2}$.

In summary, we explored top quark production through three different top decay modes, the dilepton, the lepton plus jets, and the all hadronic channel. We reviewed the current yield of top decays in these three decay modes observed by the CDF and D $\varnothing$ experiment. As was true after the discovery of the bottom quark, the top quark has been confirmed in different decay modes. In the following, we use these top event candidates to measure the fundamental quantities of the top quark: the top production cross section $\sigma_{t \bar{t}}$ and the top quark mass $m_{\mathrm{top}}$.

\subsection{The Top Production Cross Section}

The measurement of the top production cross section $\sigma_{t \bar{t}}$ is of interest for several reasons. It checks QCD calculations of top production, which have been performed by several groups, 24 a 2 and it provides an important benchmark for estimating top yields in future high statistics experiments at the Tevatron and LHC. In addition, a value of the top cross section significantly different from the QCD prediction could indicate nonstandard top prodcution or decay mechanisms.

The measurement of the top production cross section $\sigma_{t \bar{t}}$ is straight forward:

$$
\sigma_{t \bar{t}}=\frac{N_{\mathrm{obs}}-N_{\mathrm{bkg}}}{A \mathcal{L}} .
$$

The number of predicted background events $N_{\mathrm{bkg}}$ is subtracted from the number of observed top candidates $N_{\text {obs }}$ and divided by the acceptance $A$ of the sample selection and the integrated luminosity $\mathcal{L}$ of the used data set. The measurement of $\sigma_{t \bar{t}}$ has been determined in each decay channel individually as detailed in Fig. 6. The results of the different $\sigma_{t \bar{t}}$ measurements from CDF and DØ can be compared to each other and to theoretical predictions indicated by the dark band also shown in Fig. 6. The width of the theory band is given by the spread in the theoretical predictions of Laenen et al, 204 Berger et al 26 Nason et al, 国 and Catani et al.27 


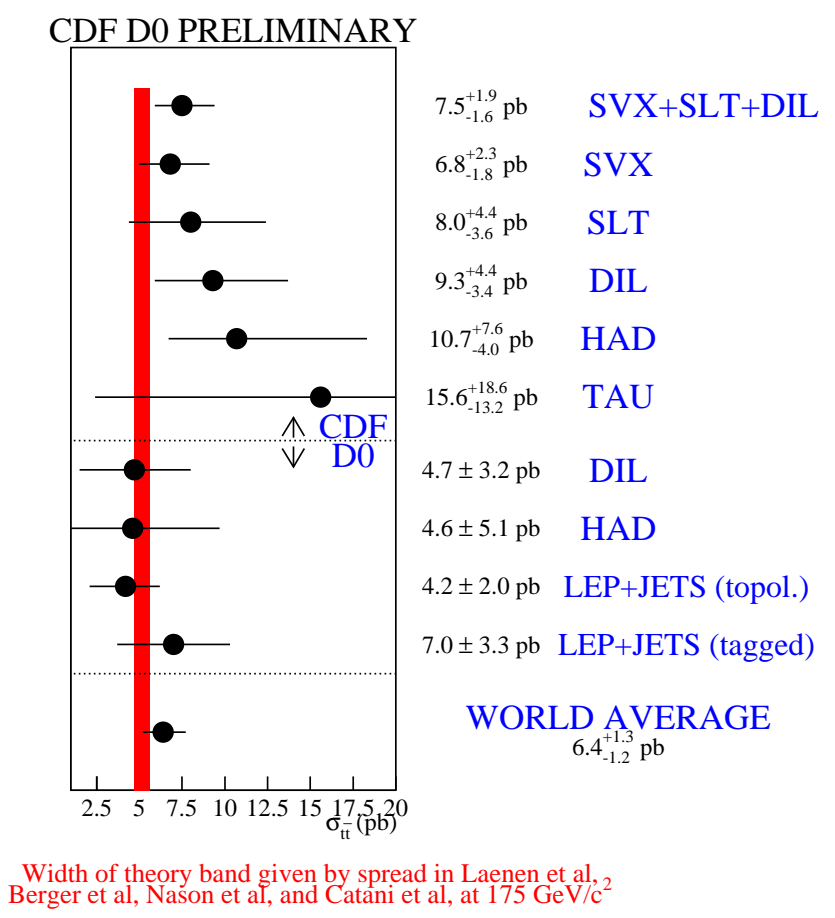

Figure 6: Summary of the CDF and D $\varnothing$ top quark production cross section measurements in different decay channels.

From all these measurements of $\sigma_{t \bar{t}}$ a world average top production cross section

$$
\sigma_{t \bar{t}}=\left(6.4_{-1.2}^{+1.3}\right) p \mathrm{~b}
$$

can be determined, which is slightly larger but in good agreement with the theoretical predictions.

\subsection{Measurement of the Top Quark Mass}

The top quark mass $m_{\text {top }}$ is a fundamental parameter in the Standard Model. A precise determination of $m_{\text {top }}$ is therefore the most important measurement of the $\mathrm{CDF}$ and $\mathrm{D} \varnothing$ experiment in Run I. The goal is to use as many decay modes as possible and to measure $m_{\text {top }}$ as accurately as possible. The use of several methods also allows cross checking of the different techniques and studying of systematic uncertainties. An incredible amount of work exploring these issues has been done by both experiments. This resulted in significantly improved measurements of $m_{\text {top }}$ since the discovery publications. 10 . The uncertainties on the top quark mass measurements by CDF and D $\varnothing$ have been improved by a factor of more than two. 


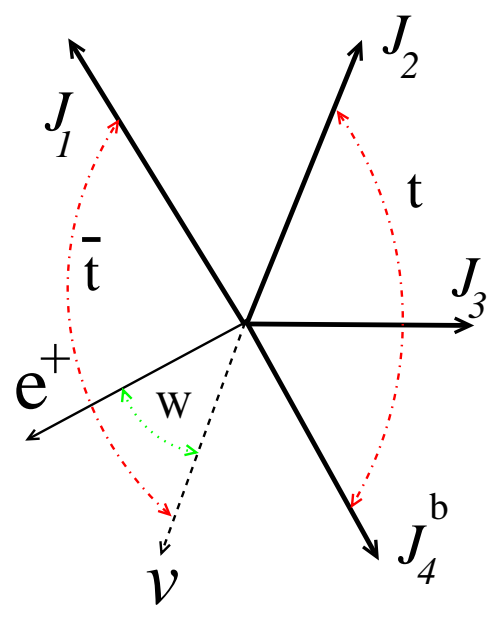

(a)

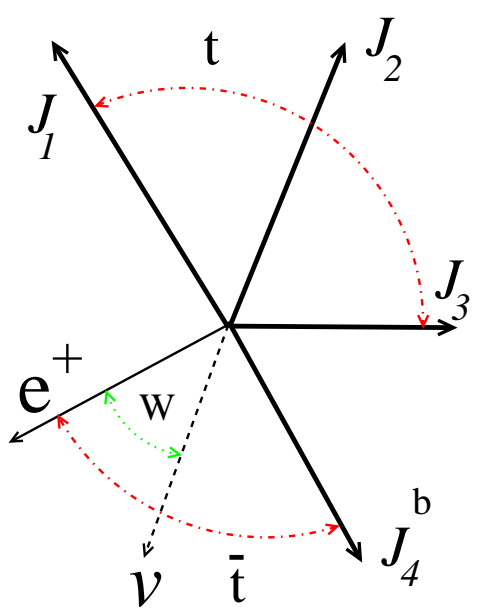

(b)

Figure 7: Illustration of different assignments of the observed objects in a $t \bar{t}$ decay to the originating partons.

In such a short time, this is a respectable amount of progress in understanding the newly discovered top quark.

The primary dataset for measuring the top quark mass is the lepton plus jets sample. This is the decay mode with the most power. The background in the all hadronic channel is too large and the statistics of the dilepton mode is too weak together with the additional complication of two missing neutrinos. Thus, the preferred method of determining $m_{\text {top }}$ by both experiments, is a constrained fit to the lepton plus 4-jet events arising from the process $t \bar{t} \rightarrow W b W \bar{b} \rightarrow \ell \nu J_{1} J_{2} J_{3}^{b} J_{4}^{\bar{b}}$. The task is to assign the observed particles, jets, and $E_{\mathrm{t}}$ in these events one-to-one to the decay products of the $t$ and $\bar{t}$. However, the problem remains that we don't know a priori how to map the observed jets to the partons from the $t \bar{t}$ decay. This is illustrated in Figure 7, where for example in Fig. 7a) jet $J_{1}$ is combined with the lepton and neutrino to originate from one top quark, while jets $J_{2}$ through $J_{4}$ are assigned to the $\bar{t}$ quark. Fig. $7 \mathrm{~b}$ ) shows another possible assignment, which might as well be the result of a $t \bar{t}$ decay. In total there are 12 possible jet-parton assignements which are reduced to six combinations if one $b$ jet is tagged. If there are two $b$ tags in the event, there are still two possible combinations from assigning the two $b$ jets to the $t$ or $\bar{t}$ quark. In additon, this combinatorics gets doubled, because there is a twofold ambiguity for $p_{z}^{\nu}$ since only the transverse component of the missing energy $\not_{\mathrm{t}}$ is reconstructed by the experiments. 
To select the best combination CDF and D $\varnothing$ use a likelihood method that exploits the many constraints in the system. Each event is fitted individually to the hypothesis that three of the jets come from one top quark through its decay into $W b$, and that the lepton, $E_{t}$, and the remaining jet come from the other top quark. In addition, $b$ tagged jets are assigned as $b$ quarks in the fit, the invariant dijet mass has to equal the $W$ boson mass, and $m_{t}=m_{\bar{t}}$ is required. CDF chooses the solution with the best fit $\chi^{2}$, while $D \varnothing$ takes a weighted average of the three best solutions, where each solution has to satisfy a minimum $\chi^{2}$ cut. The result is a distribution of the best fit top mass from each candidate event. The final value of the top mass is then extracted by fitting this distribution to a set of Monte Carlo templates from $t \bar{t}$ production and background. There are several reasons why a MC simulation of the kinematic fitting is necessary. At first, there is the need to relate the jet energies to parton energies, where the experimental smearing, predominantly in the jet energies, has to be taken into account. In addition, the four most energetic jets in the event may not be directly related to the $t \bar{t}$ decay products, if for example a hard gluon radiation produced an energetic jet. Finally, the fit solution with the lowest $\chi^{2}$ may not have the correct jet assignment.

\subsubsection{CDF Top Quark Mass Measurement}

CDF has a good signal to background ratio in the lepton plus jets channel, where one of the jets is SVX tagged. This sample of lepton plus 4-jet events with at least one SVX tag provided the original top mass measurement at CDF. 19 Recent optimization studies indicate a reduced error on $m_{\mathrm{top}}$, if the tagged events are subdivided into different tagging classes and a set of no-tagged events is added. The following four data samples are used for this optimized determination of the top quark mass: (a) events with one SVX tag (15 events), (b) events with two SVX tags (5 events), (c) events with a SLT tag but no SVX tag (14 events), and (d) events with no tag but all four leading jets having $E_{\mathrm{t}}>15 \mathrm{GeV}$ (48 events).

These four subsamples are orthogonal to each other and a top mass can be extracted from each sample individually. These top mass distributions for the four samples are shown separately in Figure $8 \mathrm{a}$ ). For each of the subsamples, an expected background fraction was estimated, and predicted top signal distributions

were obtained from Monte Carlo events at several values of $m_{\text {top }}$. A likelihood fit was performed for each subsample, which is shown in the inserts of the dis- 
(a)
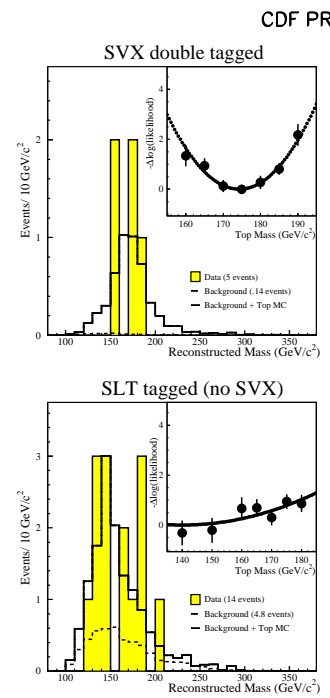

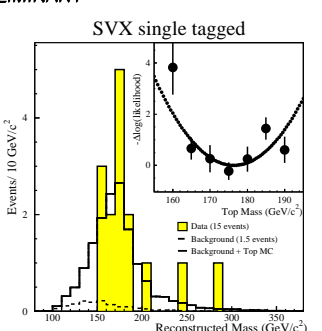

Not tagged (4 jets with $\mathrm{E}_{\mathrm{t}} \geq 15 \mathrm{GeV}$ )

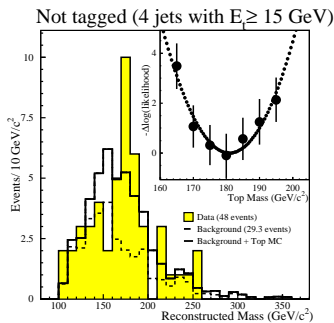

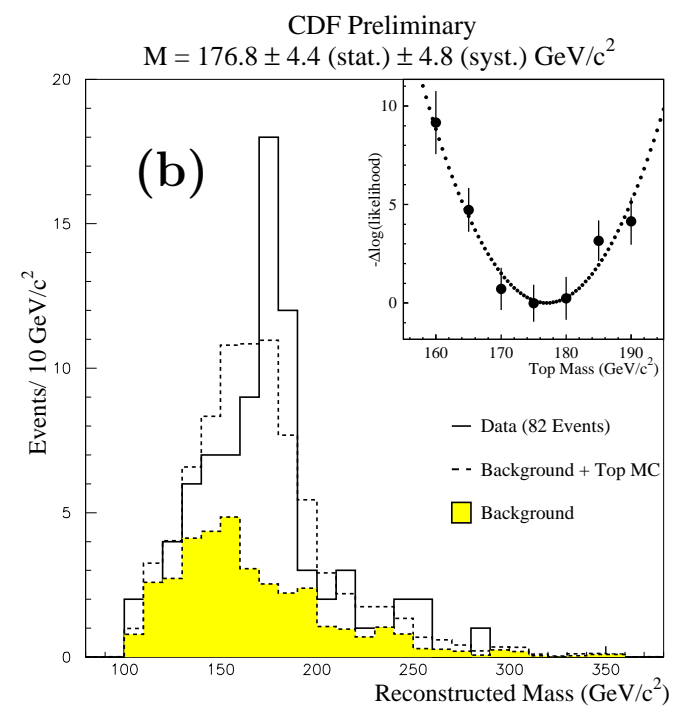

Figure 8: CDF top mass distributions: (a) For the four subsamples discussed in the text. The shaded area represents the data, while the solid line is the predicted sum of background plus expected signal. The dashed line just represents the background contribution. The inserts show the obtained likelihood function versus top mass. In (b) the combined top mass distribution is shown. The solid line represents the data, while the dashed line is the predicted sum of background plus expected signal. The shaded area represents the background contribution. The insert shows the obtained likelihood function versus top mass.

tributions in Fig. 8a). Since the likelihood values from the four subsamples are essentially independent, a combined result is obtained by taking the product of the four likelihood values. The sums of the four distributions, as well as the likelihood product versus top mass, are shown in Fig. 8b), where the solid line represents the data, while the dashed line is the predicted sum of background plus expected signal. The shaded area just represents the background contribution.

From the distribution of the likelihood product versus $m_{\text {top }}$ a top mass value of $m_{\text {top }}=(176.8 \pm 4.4 \pm 4.8) \mathrm{GeV} / c^{2}$ has been extracted. The systematic errors are detailed in Table 5. The largest contribution $\left( \pm 3.6 \mathrm{GeV} / c^{2}\right)$ comes from soft gluon radiation plus jet energy scale uncertainties in estimating the true jet energy from the observed energy. The systematic error due to hard gluon effects reflects the uncertainty in how often one of the four leading jets is associated with a hard gluon rather than directly with a parton in a top decay. All other systematic errors contribute less than $1.5 \mathrm{GeV} / c^{2}$ (see Tab. 5). 


\begin{tabular}{|c|c|}
\hline CDF Lepton plus Jets Mass Systematics & $\Delta m_{\text {top }}$ \\
\hline \hline Soft Gluon + Jet $E_{\mathrm{t}}$ Scale & $3.6 \mathrm{GeV} / c^{2}$ \\
Different MC Generators & $1.4 \mathrm{GeV} / c^{2}$ \\
Hard Gluon Effects & $2.2 \mathrm{GeV} / c^{2}$ \\
Kin. \& Likelihood Fitting Method & $1.5 \mathrm{GeV} / c^{2}$ \\
b-tagging Bias & $0.4 \mathrm{GeV} / c^{2}$ \\
Background Spectrum & $0.7 \mathrm{GeV} / c^{2}$ \\
Monte Carlo Statistics & $0.8 \mathrm{GeV} / c^{2}$ \\
\hline \hline Total & $4.8 \mathrm{GeV} / c^{2}$ \\
\hline
\end{tabular}

Table 5: Systematic uncertainties of the CDF top quark mass measurement.

\subsubsection{DØ Top Quark Mass Measurement}

As already discussed, the power to extract a top quark mass from the lepton plus 4 -jet events depends on the suppression of the background. The $\mathrm{D} \varnothing$ experiment combines four kinematic variables into one top likelihood discriminant $\mathcal{D}$, which provides a distinct separation power between top signal and background without biasing the analysis. For example, a straight cut on the total hadronic energy $H_{T}$ would push both background and signal distributions towards higher values of $m_{\text {top }}$ and make the background look like signal. The top likelihood discriminant $\mathcal{D}$ combines $E_{\mathrm{t}}$, the aplanarity $\mathcal{A}$ of the $W$ plus jets system, the fraction of the $E_{\mathrm{t}}$ of the $W$ plus jets system which is carried by the $W\left(H_{T 2}^{\prime}\right)$, and the $E_{\mathrm{t}}$ weighted RMS $\eta$ of the $W$ and jets $\left(K_{T_{m i n}}^{\prime}\right)$. Some of the distributions of these variables, as well as the combined top likelihood discriminant $\mathcal{D}$ are shown in Fig. 9a). As can be seen, there is separation between a top signal predicted from Monte Carlo (light shaded area) and background expectations (dark shaded area). After applying a cut of $\mathcal{D}>0.43,32$ events have been selected for the fit of $m_{\text {top }}$.

The distribution of the fitted top mass values is shown in Fig. 9b) as the solid histogram. The solid crosses represent the prediction for background plus expected signal, which is in good agreement with the data. The dashed crosses in Fig. 9b) show the background expectation only. A measurement of the top quark mass of $m_{\text {top }}=(168 \pm 8 \pm 8) \mathrm{GeV} / c^{2}$ has been extracted from this distribution. The insert in Fig. 9b) shows the obtained likelihood as a function of true $m_{\text {top }}$ as well as the curves used to determine the top quark mass and its statistical error. The systematic errors on the $D \varnothing$ top mass measurement are detailed in Table 6 . 

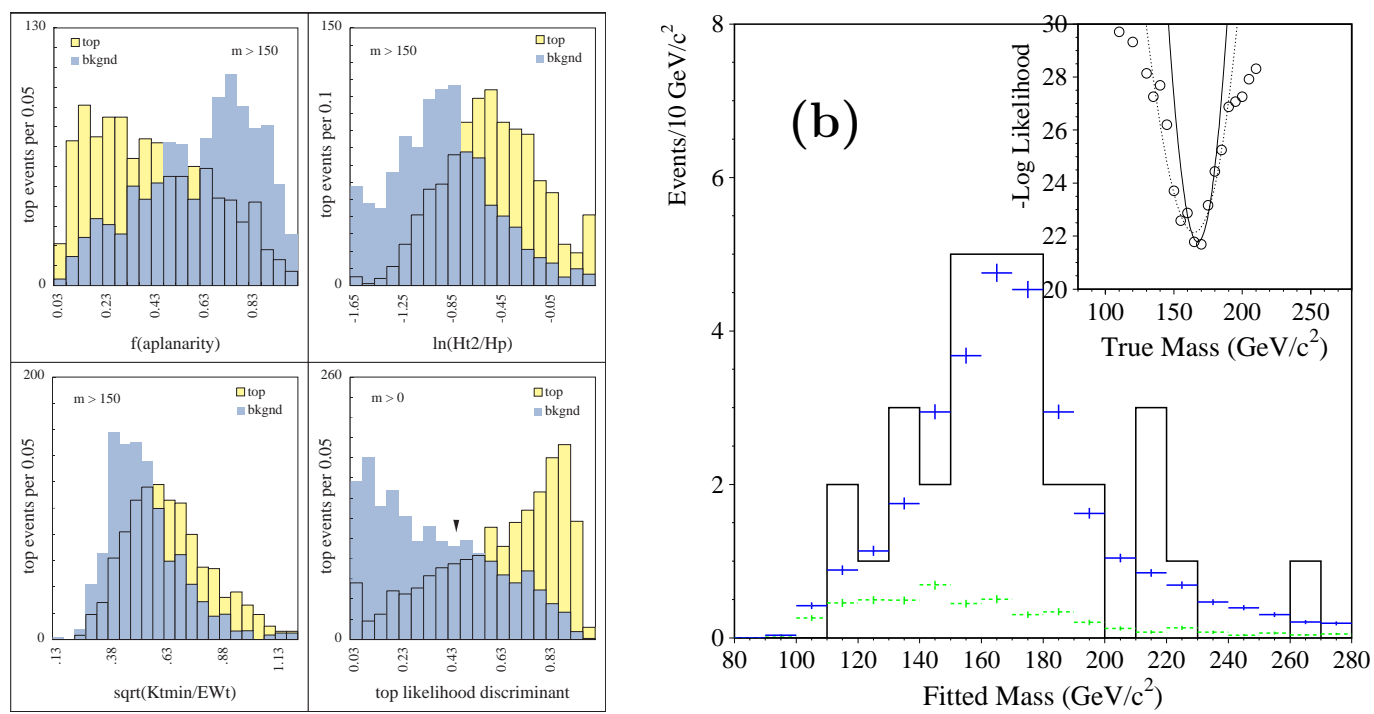

Figure 9: DØ top mass measurement: (a) Distribution of MC top (light) and background (dark) lepton plus 4-jet events for different kinematic parameters like aplanarity, $H_{T_{2}}^{\prime}, K_{T_{m i n}}^{\prime}$, and the combined top likelihood discriminat $\mathcal{D}$ (from top left to bottom right). (b) Observed (solid) top quark mass distribution, where the solid crosses represent the prediction for background plus signal. The dashed crosses are just the background expectation. The curves in the insert are used to determine the top quark mass and its statistical error.

The largest systematic uncertainty results from the jet energy correction.

\subsubsection{World Average Top Quark Mass}

An attempt can be made to combine the $\mathrm{CDF}$ and $\mathrm{D} \varnothing$ top quark mass measurements to a world average top mass. For this purpose the reported mass measurements from the lepton plus jets channel have been used. CDF measured $m_{\text {top }}=(176.8 \pm 4.4 \pm 4.8) \mathrm{GeV} / c^{2}$, while the $\mathrm{D} \varnothing$ measurement results in

\begin{tabular}{|c|c|}
\hline DØ Lepton plus Jets Mass Systematics & $\Delta m_{\text {top }}$ \\
\hline \hline Jet Energy Correction & $7.3 \mathrm{GeV} / c^{2}$ \\
Monte Carlo Model & $3.3 \mathrm{GeV} / c^{2}$ \\
Fitting Method & $2.0 \mathrm{GeV} / c^{2}$ \\
\hline \hline Total & $8 \mathrm{GeV} / c^{2}$ \\
\hline
\end{tabular}

Table 6: Systematic uncertainties of the D $\varnothing$ top quark mass measurement. 
$m_{\text {top }}=(169 \pm 8 \pm 8) \mathrm{GeV} / c^{2}$. In the determination of the world average top mass the conservative assumption has been made that all the systematic errors, except for the energy scale, the $b$ tagging bias, and the Monte Carlo statistics, are fully correlated between both experiments. This results in a world average top quark mass of

$$
m_{\text {top }}=(175.0 \pm 3.9 \pm 4.5) \mathrm{GeV} / c^{2} .
$$

It is worthwhile to note that knowing the top quark mass with a combined statistical and systematic error of $\pm 6 \mathrm{GeV} / c^{2}$ (a $3.4 \%$ relative error) is a big accomplishment of both Tevatron experiments in Run I.

\subsubsection{Summary of Top Quark Mass Measurements}

A summary of all top quark mass measurements performed by CDF and D $\varnothing$ is given in Fig. 10. Top mass measurements from other top decay modes like the dilepton channel or the all hadronic channel are also shown. They are in good agreement with the measurement from the lepton plus 4-jet samples, which have been discussed in the previous sections in more detail. This decay mode represents the most precise determination of the top mass for each experiment.

The knowledge of the top quark mass plays an important role in calculations of radiative corrections that relate electroweak parameters. For example, higher order radiative corrections relate the $W$ boson mass $m_{W}$ to the top quark mass $m_{\text {top }}$. This relationship also depends on the mass of the Higgs boson $m_{\text {Higgs }}$, which can also participate in these higher order loops. The relationship between $M_{W}$ and $m_{\text {top }}$ is displayed in Fig. 11. The well known dependence on the Higgs mass is shown through the different bands for several assumptions of $m_{\mathrm{Higgs}}$. Although $m_{W}$ and $m_{\text {top }}$ are precisely known, the sensitivity on the Higgs boson mass is still too poor. A precise measurement of the top quark mass together with $m_{W}$ is therefore a high priority of both collider experiments in Run II. 


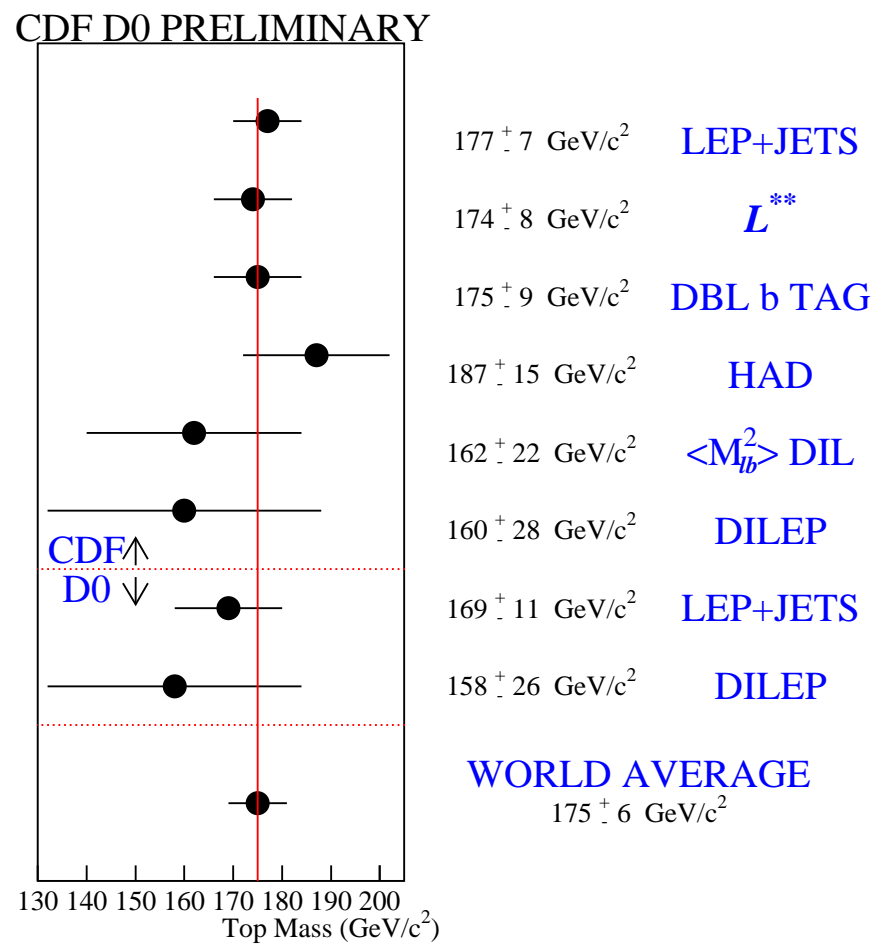

Figure 10: Summary of the CDF and D $\emptyset$ top quark mass measurements in different decay channels.

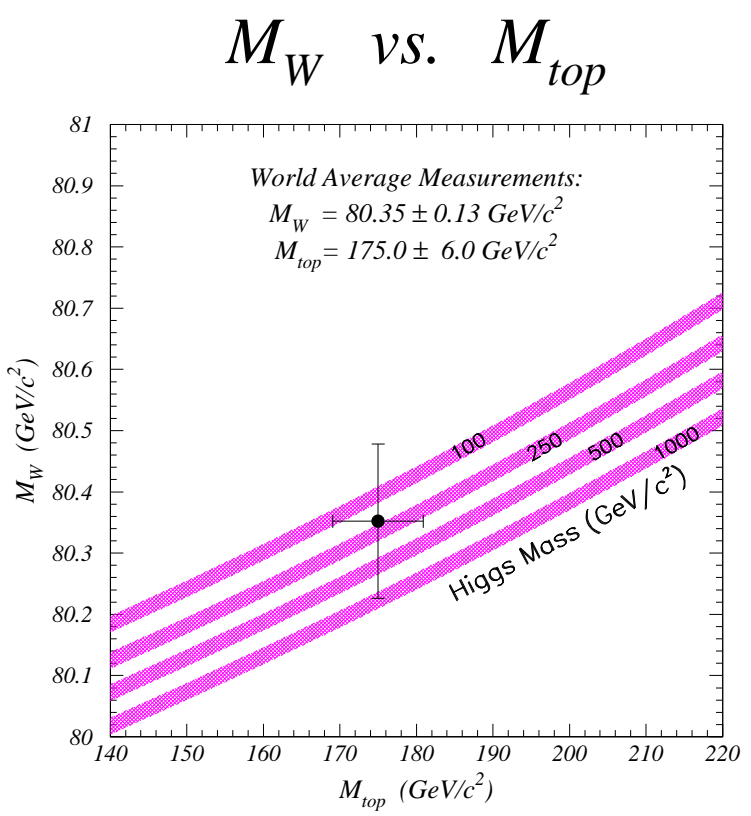

Figure 11: Relation between the top quark mass and the $W$ boson mass. The dependence on the Higgs mass is shown through the different bands for several assumptions of $m_{\mathrm{Higgs}}$. 


\section{$3 \quad B$ Physics at a Hadron Collider}

The principal interest in studying $B$ hadrons in the context of the Standard Model arises from the fact that $B$ hadron decays provide valuable information on the weak mixing matrix, the Cabibbo-Kobayashi-Maskawa (CKM) matrix.28 In fact, $B$ decays determine five of the nine CKM matrix elements: $V_{c b}, V_{u b}, V_{t d}, V_{t s}$, and $V_{t b}$. The future interest in $B$ physics certainly lies in the study of $C P$ violation in the system of neutral $B$ mesons, which will be discussed further in Sec. 4.2.

Traditionally, $B$ physics has been the domain of $e^{+} e^{-}$machines, but already the UA 1 collaboration has shown that $B$ physics is feasible at a hadron collider.29 However, the combination of a better mass resolution and vertex detection enables the CDF experiment to perform a broader $B$ physics program. The D $\varnothing$ experiment has also published several $B$ physics results, 30 but due to the lack of a precision momentum measurement of charged particles within a magnetic field and the absence of a precision micro vertex detector, $\mathrm{D} \varnothing$ is not ideally suited to do $B$ physics. Since we concentrate in this presentation on recent results from $B$ hadron lifetimes and time dependent $B^{0} \bar{B}^{0}$ oscillations, we shall report only on measurements from the CDF experiment.

One advantage of $B$ physics at a hadron collider compared to an $e^{+} e^{-}$machine at the $\Upsilon(4 S)$ is that all $B$ hadron species are produced. Another advantage in doing $B$ physics at a hadron colliders can be seen by comparing the $B$ production cross section, which is about $1 \mathrm{nb}$ at the $\Upsilon(4 S)$, while at the $Z^{0}$ pole $\sigma(B \bar{B}) \approx 6 \mathrm{nb}$. However, at the Tevatron the $b$ quark production cross section is quite large with $\sigma_{b} \sim 50 \mu \mathrm{b}$ within the central region of rapidity less than 1 $(|\cos \theta|<1)$. This is a huge cross section which resulted in about $5 \cdot 10^{9} b \bar{b}$ pairs being produced in Run I within the Tevatron detectors. But the total inelastic cross section is still about three orders of magnitude larger. This puts certain requirements on the trigger system to find $B$ decay products. In addition the total integrated $b$ quark production cross section is a rapidly falling cross section, which drops by about two orders of magnitude for a $b$ quark $p_{\mathrm{t}}^{b}$ greater than about $8 \mathrm{GeV} / c$ compared to $p_{\mathrm{t}}^{b}$ greater than about $20 \mathrm{GeV} / c$. This means, in terms of trigger thresholds for $b$ decay products, one likes to go as low as possible in $p_{\mathrm{t}}$ in order to increase the amount of recorded $B$ triggers. Of course the experiment's DAQ bandwidth is the limiting factor.

All $B$ physics triggers at CDF are based on leptons. Dilepton and single lepton 

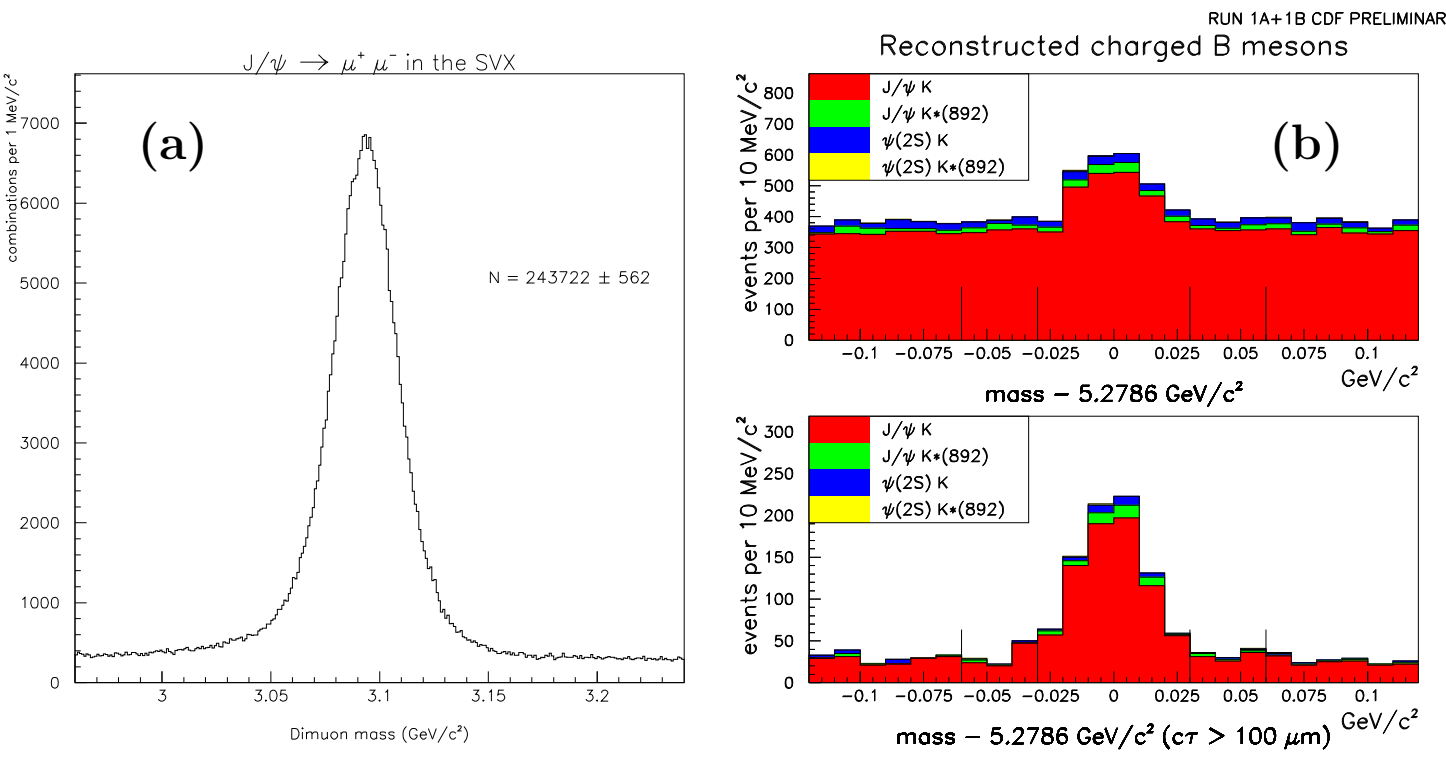

Figure 12: Invariant mass distribution of (a) dimuon trigger in the $J / \psi$ mass region and (b) $B^{+} \rightarrow J / \psi K^{+}$candidates without (top) and with a $c \tau>100 \mu \mathrm{m}$ requirement (bottom).

triggers both exist. Exploiting the steeply falling $b$ quark production cross section, CDF was able to maintain low $p_{\mathrm{t}}$ thresholds throughout Run I without increasing the experiments deadtime during data taking.

CDF's dilepton triggers consist of a dimuon trigger with $p_{\mathrm{t}}>2 \mathrm{GeV} / c$ for both muon legs, and an $e \mu$ trigger with $p_{\mathrm{t}}^{\mu}>3 \mathrm{GeV} / c$ and $E_{\mathrm{t}}^{e}>5 \mathrm{GeV}$. The dimuon trigger is the source of CDF's $J / \psi$ sample. Both dilepton trigger samples are also starting points for $B$ mixing analyses. The thresholds for the single lepton triggers are higher with $p_{\mathrm{t}}>7.5 \mathrm{GeV} / c$ for muons and $E_{\mathrm{t}}>8 \mathrm{GeV}$ for electrons. Analyses involving semileptonic $B$ decays are based on these single lepton datasets. The given $p_{\mathrm{t}}$ thresholds are representative for Run Ib but similar for Run Ia.

The basis of CDF's $B$ physics program are the good tracking and vertexing capabilities of the CTC and the SVX. This is demonstrated in Figure 12. The invariant dimuon mass from CDF's dimuon trigger sample is shown in Fig. 12a). From all Run I data a prominent signal of about 240,000 J/ $\psi$ candidates with both muons in the SVX can be found on low background. The mass resolution of the observed signal is about $16 \mathrm{MeV} / c^{2}$. One handle to reduce backgrounds with the help of CDF's silicon vertex detector is demonstrated in Fig. 12b). Here, $J / \psi$ candidates are paired with another track in the event, which is assumed to be a kaon, in order to fully reconstruct $B^{+} \rightarrow J / \psi K^{+}$decays. The top plot shows 
the invariant $J / \psi K^{+}$mass distribution, where a $B^{+}$signal is observed on a large background. This background is drastically reduced after a displaced $B$ vertex with e.g. $c \tau>100 \mu \mathrm{m}$ as measured in the SVX is required (see Fig. 12b).

\section{1 $B$ Hadron Lifetimes}

The lifetimes of $B$ hadrons are fundamental properties of these particles and can be used to test theoretical models of heavy flavour decays. Predictions for $B$ hadron

lifetimes and their ratios have been made by several groups. 31.32 Bigi et al. 31 predicts the charged $B$ lifetime to be longer than the $B^{0}$ lifetime by about $5 \%$

$$
\frac{\tau\left(B^{+}\right)}{\tau\left(B^{0}\right)} \simeq 1.0+0.05 \cdot \frac{f_{B}^{2}}{(200 \mathrm{MeV})^{2}},
$$

and expects the lifetime of the $\Lambda_{b}^{0}$ baryon to be shorter than $\tau\left(B^{0}\right)$ with a ratio of $\tau\left(\Lambda_{b}^{0}\right) / \tau\left(B^{0}\right)$ not smaller than about 0.9. On the other hand using the heavy quark expansion, Neubert 32 obtains:

$$
\begin{aligned}
\frac{\tau\left(B^{+}\right)}{\tau\left(B^{0}\right)} & =1.0+\mathcal{O}\left(1 / m_{b}^{3}\right) \\
\frac{\tau\left(\Lambda_{b}^{0}\right)}{\tau\left(B^{0}\right)} & =0.98+\mathcal{O}\left(1 / m_{b}^{3}\right)
\end{aligned}
$$

where the estimate for $\tau\left(\Lambda_{b}^{0}\right) / \tau\left(B^{0}\right)$ includes corrections that arise at order $1 / m_{b}^{2}$. Although these ratios might appear to be close to unity, Neubert argues that the $1 / m_{b}^{3}$ corrections might be large due to phase space enhancement from effects involving the $u, d$ spectator quark. He concludes that theoretical uncertainties allow a lifetime ratio in the range between 0.8 and 1.2 .

This subject is controversal, and best solved by precisely measuring all the $B$ hadron lifetimes. As we shall see, the uncertainties on the individual $B$ lifetimes have reached a level of a few percent. In the following, we review the latest $B$ hadron lifetime results from CDF and also compare them to other experiments.

\subsection{1 $B^{+}$and $B^{0}$ Lifetimes with Fully Reconstructed $B$ Mesons}

The analysis principle for the $B$ lifetime measurement using fully reconstructed $B^{+}$ and $B^{0}$ mesons is as follows. All Run I dimuons forming a $J / \psi$ candidate, as shown in Fig. 12 a), are used. All possible decay modes $B \rightarrow \Psi \mathbf{K}$ have been investigated, where $\Psi$ is mainly a $J / \psi$ but can also be a $\psi(2 S) \rightarrow \mu \mu$. K represents the different 

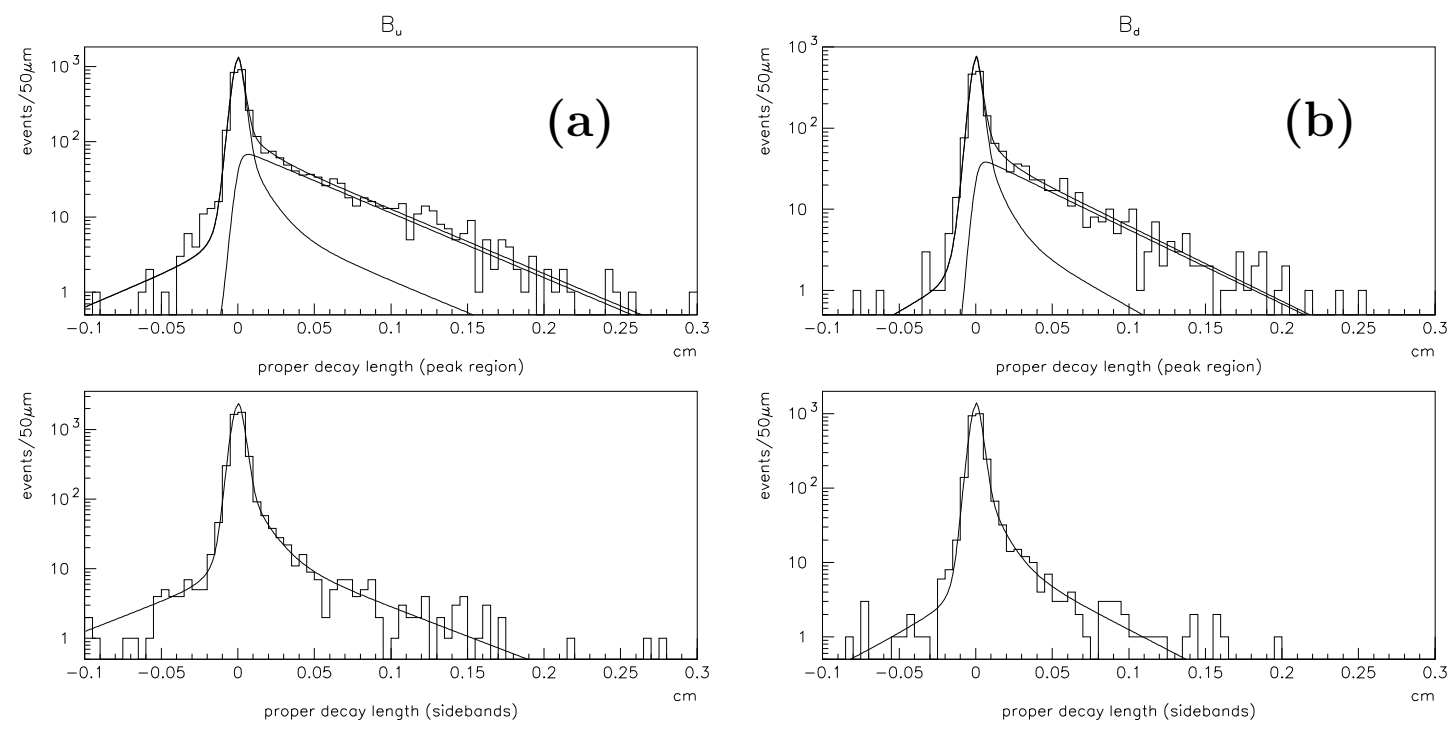

Figure 13: Proper time distributions of (a) charged and (b) neutral $B$ mesons, which were fully reconstructed through $B \rightarrow \Psi \mathbf{K}$. The bottom plots represent the background $c \tau$ distributions as obtained from the $B$ sidebands.

kaon states $K^{+}$and $K^{*}(892)^{+}$for the charged $B$ lifetime measurement, as well as $K_{S}^{0}$ and $K^{*}(892)^{0}$ for the $\tau\left(B^{0}\right)$ determination. The vertex and mass constrained $J / \psi$ candidates are vertexed with the $\mathbf{K}$ candidates yielding the two-dimensional decay length $L_{\mathrm{xy}}$. Together with the known $B$ transverse momentum $p_{\mathrm{t}}$, the proper time distributions, shown in Fig. 13 for (a) charged and (b) neutral $B$ candidates, are obtained. The bottom $c \tau$ distributions represent the background as obtained by fitting the $B$ sideband regions to a gaussian with exponential tails. Using this background shape, an unbinned likelihood fit of the signal, which is assumed to be an exponential convoluted with a Gaussian, is performed. The following lifetimes are obtained:

$$
\begin{gathered}
\tau\left(B^{+}\right)=(1.68 \pm 0.07 \pm 0.02) p \mathrm{~s} \\
\tau\left(B^{0}\right)=(1.58 \pm 0.09 \pm 0.02) p \mathrm{~s} \\
\tau\left(B^{+}\right) / \tau\left(B^{0}\right)=1.06 \pm 0.07 \pm 0.01 .
\end{gathered}
$$

The exclusive $B$ lifetime measurement is still statistics dominated. One way to increase the number of $B$ candidates is to not fully reconstruct the $B$ meson. This is done in the semi-exclusive analysis described in the next section. 

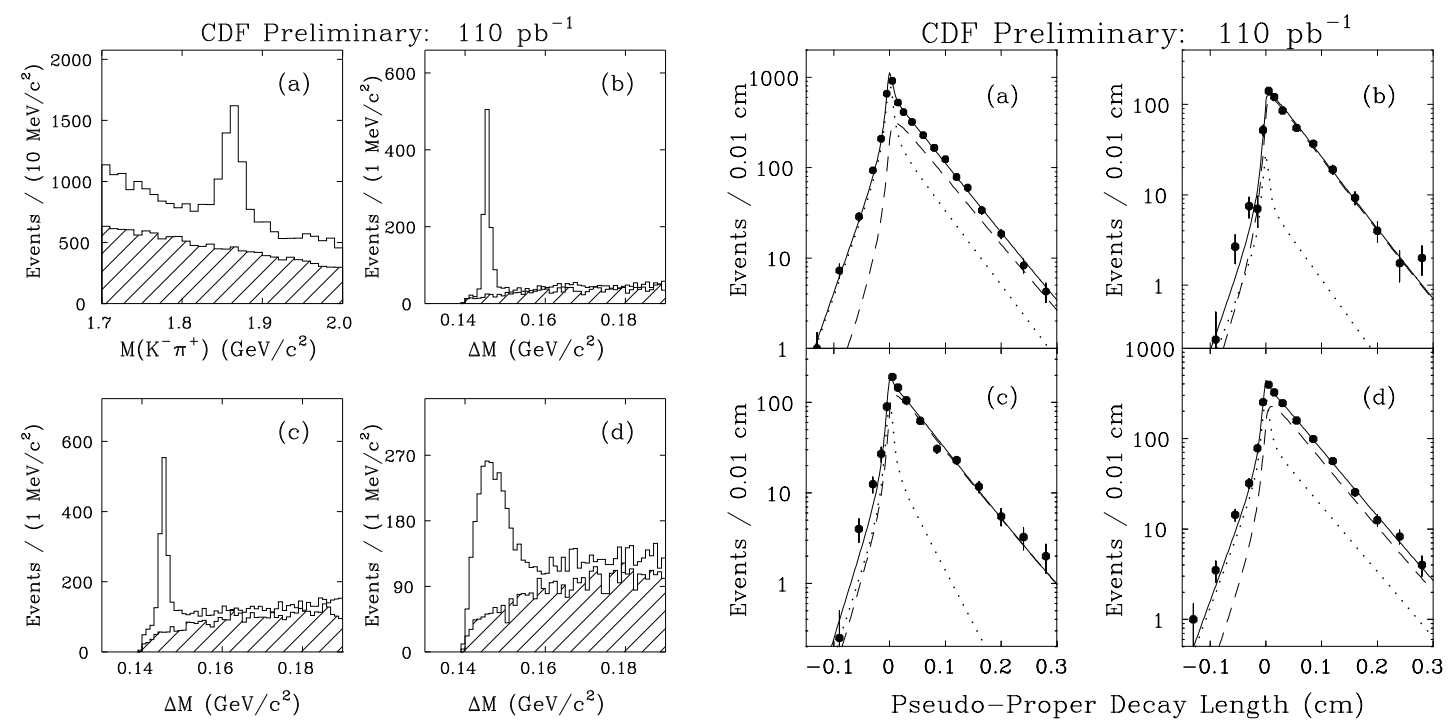

Figure 14: Invariant mass distribution of $D^{(*)}$ candidates (left hand side) and proper time distributions (right hand side) from the $B$ lifetime analysis using partially reconstructed $B \rightarrow D^{(*)} \ell X$ decays with (a) $D^{0} \rightarrow K^{-} \pi^{+}$, where the $D^{0}$ is not from a $D^{*+}$, (b) $D^{*+} \rightarrow D^{0} \pi^{+}, D^{0} \rightarrow K^{-} \pi^{+}$, (c) $D^{*+} \rightarrow D^{0} \pi^{+}, D^{0} \rightarrow$ $K^{-} \pi^{+} \pi^{+} \pi^{-}$, and (d) $D^{*+} \rightarrow D^{0} \pi^{+}, D^{0} \rightarrow K^{-} \pi^{+} \pi^{0}$.

\subsection{2 $B^{+}$and $B^{0}$ Lifetimes with Partially Reconstructed $B$ Mesons}

The $B$ lifetime analysis using partially reconstructed $B$ mesons exploits the semileptonic decays $B \rightarrow D^{(*)} \ell X$ and starts with the single lepton trigger data. In a cone around the trigger electron or muon, $D^{(*)}$ meson candidates are reconstructed through their decay modes:

1. $D^{0} \rightarrow K^{-} \pi^{+}$, where the $D^{0}$ is not from a $D^{*+}$,

2. $D^{*+} \rightarrow D^{0} \pi^{+}, D^{0} \rightarrow K^{-} \pi^{+}$,

3. $D^{*+} \rightarrow D^{0} \pi^{+}, D^{0} \rightarrow K^{-} \pi^{+} \pi^{+} \pi^{-}$,

4. $D^{*+} \rightarrow D^{0} \pi^{+}, D^{0} \rightarrow K^{-} \pi^{+} \pi^{0}$, where the $\pi^{0}$ is not reconstructed.

The $D^{(*)}$ candidates are intersected with the lepton to find the $B$ decay vertex. Since the $B$ meson is not fully reconstructed, its $c \tau_{B}$ cannot be directly determined. A correction has to be applied to scale from the $D^{(*)} \ell$ momentum to $p_{\mathrm{t}}(B)$. This $\beta \gamma$ correction is obtained from a Monte Carlo simulation.

The final $D^{(*)}$ candidates can be seen on the left hand side of Fig. 14 for (a) $D^{0} \rightarrow K^{-} \pi^{+}$, where the $D^{0}$ is not from a $D^{*+}$, (b) $D^{*+} \rightarrow D^{0} \pi^{+}, D^{0} \rightarrow K^{-} \pi^{+}$, (c) $D^{*+} \rightarrow D^{0} \pi^{+}, D^{0} \rightarrow K^{-} \pi^{+} \pi^{+} \pi^{-}$, and (d) $D^{*+} \rightarrow D^{0} \pi^{+}, D^{0} \rightarrow K^{-} \pi^{+} \pi^{0}$. 
Although the resolution of the $D^{*+}$ mass peak is worse in mode (d) compared to the other channels, it is still good enough to be used in this analysis. Note, the charm signals in Fig. 14 are quite clean and rather competitive with $D^{(*)}$ signals found at $e^{+} e^{-}$machines. This demonstrates the feasibility of $B$ physics in a hadron collider environment without using $J / \psi$ 's.

The obtained lifetime distributions from $\ell^{+} \bar{D}^{0}$ and $\ell^{+} D^{*-}$ are used to determine the individual $B^{+}$and $B^{0}$ lifetimes. A $\ell^{+} \bar{D}^{0}$ combination usually originates from a charged $B$ meson while $\ell^{+} D^{*-}$ comes from a $B^{0}$. This simple picture is complicated by the existence of $D^{* *}$ states which are the source of $\bar{D}^{0}\left(D^{*-}\right)$ mesons that originate from a decay $B^{0} \rightarrow D^{* *-} \ell^{+}, D^{* *-} \rightarrow \bar{D}^{0} X$ $\left(B^{+} \rightarrow \bar{D}^{* * 0} \ell^{+}, \bar{D}^{* * 0} \rightarrow D^{*-} X\right)$. This cross talk from $D^{* *}$ resonances has been decomposed using Monte Carlo. A combined lifetime fit, as shown on the righthand side of Fig. 14, yields the following $B$ lifetimes:

$$
\begin{gathered}
\tau\left(B^{+}\right)=(1.64 \pm 0.06 \pm 0.05) p \mathrm{~s} \\
\tau\left(B^{0}\right)=(1.48 \pm 0.04 \pm 0.05) p \mathrm{~s} \\
\tau\left(B^{+}\right) / \tau\left(B^{0}\right)=1.11 \pm 0.06 \pm 0.03 .
\end{gathered}
$$

\subsection{3 $B^{+}$and $B^{0}$ Lifetime Comparison}

A comparison of the $\mathrm{CDF} B^{+}$and $B^{0}$ lifetime measurements with other experiments, as presented at the 28th International Conference on High Energy Physics, Warsaw, Poland (ICHEP'96) 33 can be found in Figures 15 and 16, respectively. A comparison of the CDF lifetime ratio measurements is presented in Figure 17. The $B$ lifetime analysis using partially reconstructed $B \rightarrow D^{(*)} \ell X$ decays is a new result comprising the full Run I statistics and updates the 1992-93 measurement. These new measurements have not yet been available at the time of the ICHEP'96 conference and have been added to Figures 15 through 17. This comparison shows that the CDF $B$ lifetime measurements are competitive with the results from the $Z$ pole at LEP and SLC.

The combination of the presented $B$ lifetime results using fully and partially reconstructed $B$ decays yields the following combined CDF $B$ lifetime average:

$$
\begin{array}{r}
\tau\left(B^{+}\right)=(1.66 \pm 0.05) p \mathrm{~s} \\
\tau\left(B^{0}\right)=(1.52 \pm 0.06) p \mathrm{~s} \\
\tau\left(B^{+}\right) / \tau\left(B^{0}\right)=1.09 \pm 0.05 .
\end{array}
$$




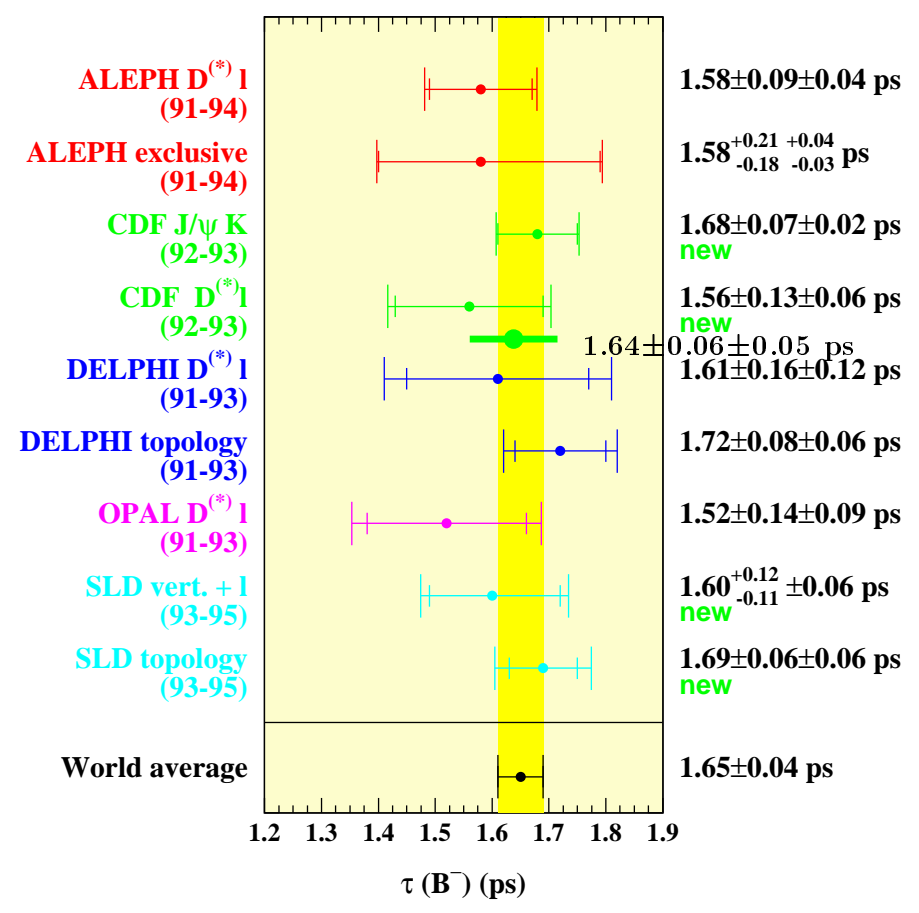

Figure 15: Comparison of the CDF $B^{+}$lifetime measurements with other experiments.

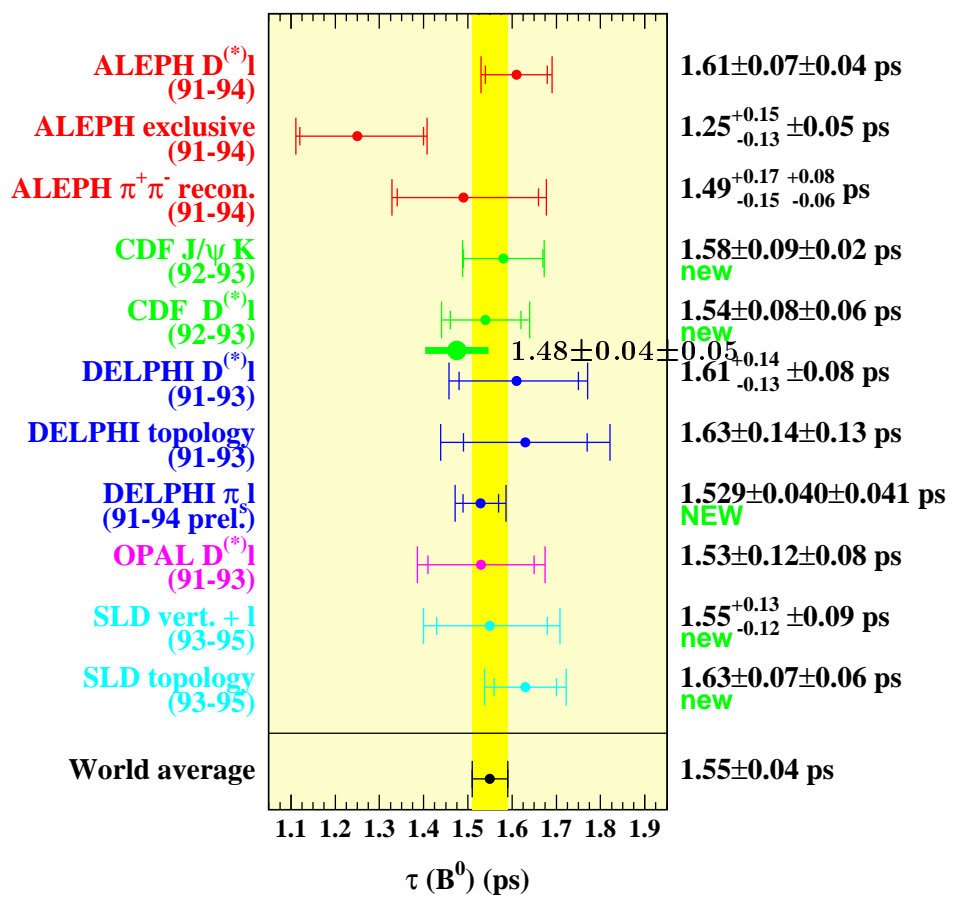

Figure 16: Comparison of the $\mathrm{CDF} B^{0}$ lifetime measurements with other experiments. 


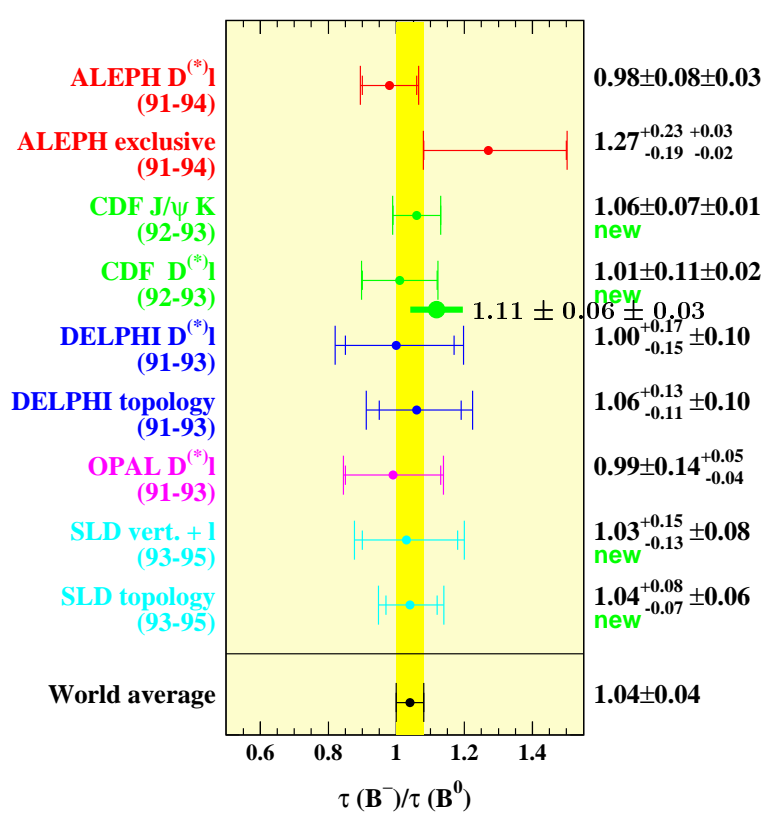

Figure 17: Comparison of the $\mathrm{CDF} \tau\left(B^{+}\right) / \tau\left(B^{0}\right)$ lifetime ratio measurements with other experiments.

The error is the sum of statistical and systematic errors, where the correlated systematic errors are taken into account. It appears that the lifetime ratio measurement is different from unity by almost two standard deviations.

\subsection{4 $B$ Lifetimes: $B_{S}^{0}$ Meson}

The lifetime of the $B_{S}^{0}$ meson is measured at CDF using the semileptonic decay $B_{S}^{0} \rightarrow D_{S}^{-} \ell^{+} \nu X$, where the $D_{S}^{-}$is reconstructed through its decay mode $D_{S}^{-} \rightarrow$ $\phi \pi^{-}, \phi \rightarrow K^{+} K^{-}$. The analysis starts again with the single lepton $(e, \mu)$ trigger data searching for $D_{S}^{-} \rightarrow \phi \pi^{-}$candidates in a cone around the lepton. The $D_{S}^{-}$ candidates are intersected with the lepton to find the $B_{S}^{0}$ decay vertex from where the analysis follows the description of the $B$ lifetime measurement using partially reconstructed $B$ mesons (see Sec. 3.1.2). A signal of $(254 \pm 21) D_{S}^{-} \ell^{+}$candidates is found as shown in Fig. 18a), where the $\phi \pi^{-}$invariant mass distribution for right $\operatorname{sign} D_{S}^{-} \ell^{+}$combinations is plotted. The shaded histogram shows the wrong sign $D_{S}^{-} \ell^{-}$distribution. Using these events, the $B_{S}^{0}$ meson lifetime is determined to be

$$
\tau\left(B_{S}^{0}\right)=\left(1.37_{-0.12}^{+0.14} \pm 0.04\right) p s .
$$

A comparison of the $\mathrm{CDF} B_{S}^{0}$ lifetime measurement with other experiments at LEP and SLC can be found in Fig. 18b). 

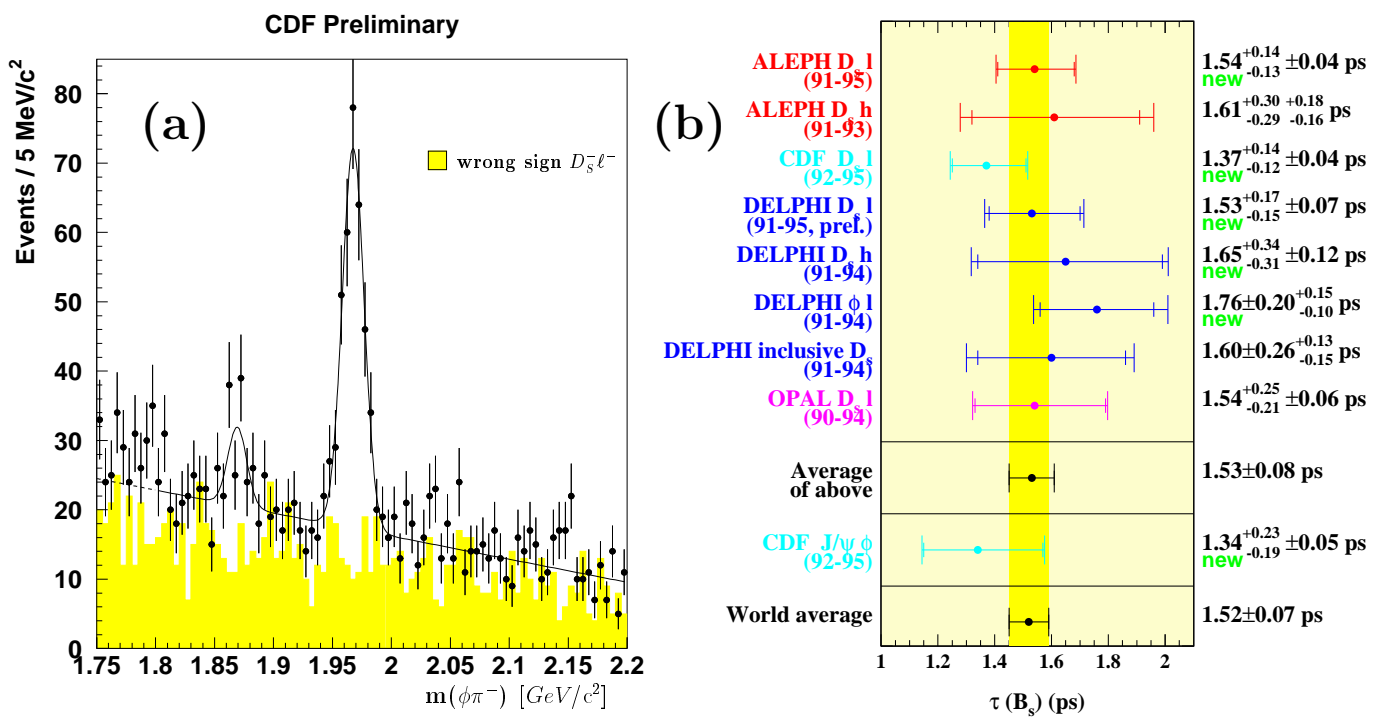

Figure 18: $B_{S}^{0}$ lifetime measurement: (a) Invariant $\phi \pi^{-}$mass distribution for right sign $D_{S}^{-} \ell^{+}$combinations. The shaded histogram shows the wrong sign distribution. (b) Comparison of the $\mathrm{CDF} B_{S}^{0}$ lifetime measurement with other experiments.

\subsection{5 $B$ Lifetimes: $\Lambda_{b}^{0}$ Baryon}

The analysis principle for the $\Lambda_{b}^{0}$ lifetime measurement is very similar to the $B_{S}^{0}$ lifetime analysis at CDF. The $\Lambda_{b}^{0}$ baryon is reconstructed through the semileptonic decay $\Lambda_{b}^{0} \rightarrow \Lambda_{c}^{+} \ell^{-} \nu X$, with the subsequent decay $\Lambda_{c}^{+} \rightarrow p K^{-} \pi^{+}$. The analysis again uses the single lepton trigger data searching for $\Lambda_{c}^{+} \rightarrow p K^{-} \pi^{+}$candidates in a cone around the lepton. The $\Lambda_{c}^{+}$candidates are intersected with the lepton to find the $\Lambda_{b}^{0}$ decay vertex. A signal of $(197 \pm 25) \Lambda_{c}^{+}$candidates is obtained as shown in Fig. 19a), where the $p K \pi$ invariant mass distribution for right sign $\Lambda_{c}^{+} \ell^{-}$combinations is plotted. The shaded histogram shows the wrong sign $\Lambda_{c}^{-} \ell^{-}$ distribution. Using these events, the $\Lambda_{b}^{0}$ lifetime is determined to be

$$
\tau\left(\Lambda_{b}^{0}\right)=(1.32 \pm 0.15 \pm 0.07) p \mathrm{~s}
$$

A comparison of the $\mathrm{CDF} \Lambda_{b}^{0}$ lifetime measurement with LEP results can be seen in Fig. 19b). The CDF $\Lambda_{b}^{0}$ lifetime is competitive with the LEP measurements in precision, but tend to be longer compared to the LEP results. 

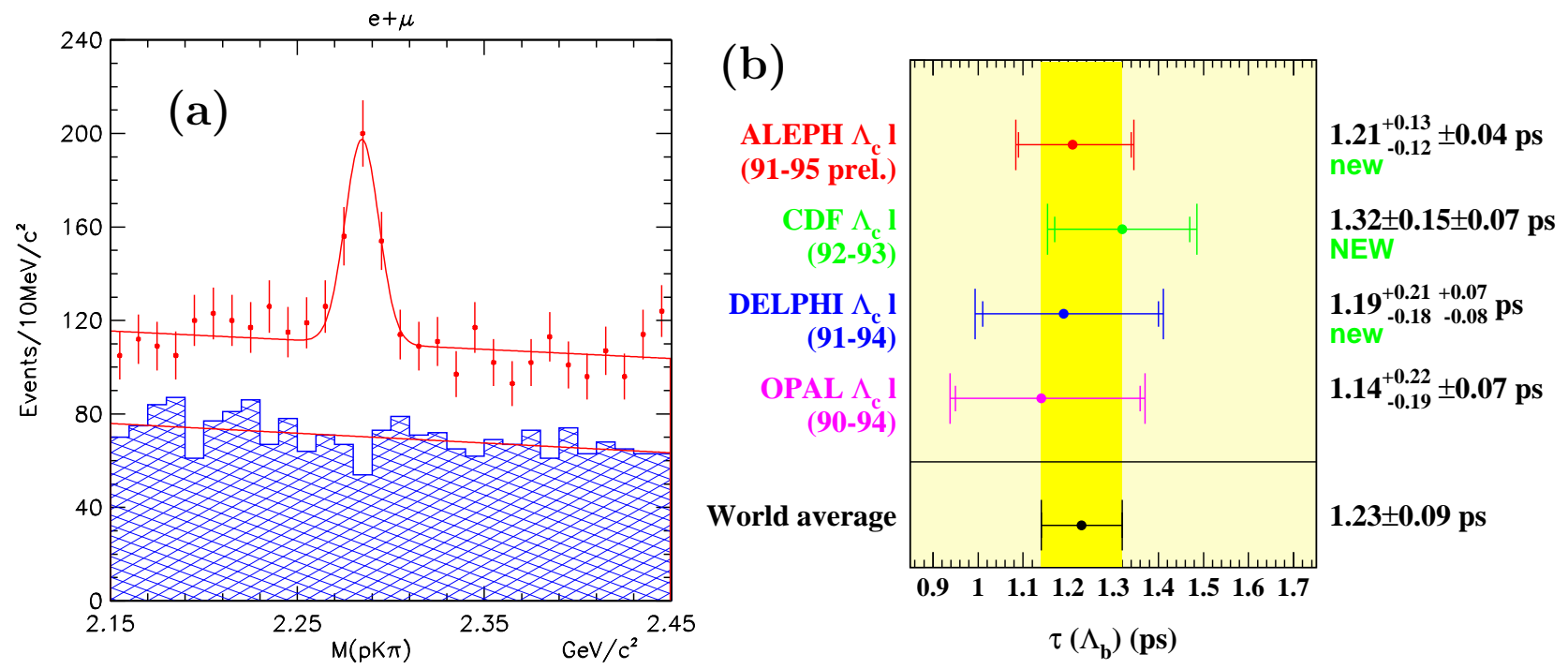

Figure 19: $\Lambda_{b}^{0}$ lifetime measurement: (a) Invariant $p K \pi$ mass distribution for right sign $\Lambda_{c}^{+} \ell^{-}$combinations. The shaded histogram shows the wrong sign distribution. (b) Comparison of the CDF $\Lambda_{b}^{0}$ lifetime measurement with LEP results.

\subsubsection{B Lifetimes: Summary}

A summary of the $B$ lifetime measurements at CDF is given in Table 7. As we have seen, CDF's $B$ lifetime results are very competitive with the LEP and SLC measurements where a precision of a few percent is reached. Although CDF's measurement of the $B^{+} / B^{0}$ lifetime ratio appears to be different from unity by almost two standard deviations, the precision is still not yet sufficient to distinguish between theoretical approaches. The $\Lambda_{b}^{0}$ lifetime lies closer to the $B^{0}$ lifetime at CDF with a ratio of $\tau\left(\Lambda_{b}^{0}\right) / \tau\left(B^{0}\right)=0.87 \pm 0.11$, while the LEP experiments report a ratio of $0.78 \pm 0.04 .3$ Theory favours the value for this ratio to be closer to 0.9-1.0 in good agreement with the CDF measurement.

\begin{tabular}{|rl|}
\hline CDF B Lifetime Summary \\
\hline \hline$\tau\left(B^{+}\right)=(1.66 \pm 0.05) p \mathrm{~s}$ \\
$\tau\left(B^{0}\right)=(1.52 \pm 0.06) p \mathrm{~s}$ \\
$\tau\left(B^{+}\right) / \tau\left(B^{0}\right)=1.09 \pm 0.05$ \\
\hline$\tau\left(B_{S}^{0}\right)=(1.37 \pm 0.14) p \mathrm{~s}$ \\
$\tau\left(\Lambda_{b}^{0}\right)=(1.32 \pm 0.17) p \mathrm{~s}$ \\
\hline
\end{tabular}

Table 7: Summary of CDF $B$ hadron lifetime results. 


\section{2 $B \bar{B}$ Oscillations}

In the Standard Model $B \bar{B}$ mixing occurs through the electroweak box diagram, where the dominant contribution is through the top quark loop. The size of the oscillation is expressed in terms of the mixing parameter $x=\Delta m / \Gamma$, where $\Delta m$ is the difference in mass between the two $B$ meson eigenstates and $\Gamma$ refers to the average lifetime of both $B$ states $\tau_{B}=\hbar / \Gamma$. For a beam initially pure in $B^{0}$ mesons (at $t=0$ ), the numbers of $B^{0}$ and $\bar{B}^{0}$ mesons at proper time $t, N(t)_{B^{0} \rightarrow B^{0}}$ and $N(t)_{B^{0} \rightarrow \bar{B}^{0}}$, respectively, are given by:

$$
\begin{aligned}
N(t)_{B^{0} \rightarrow B^{0}} & =\frac{1}{2 \tau_{B}} e^{-t / \tau_{B}}\left(1+\cos \Delta m_{d} t\right) \\
N(t)_{B^{0} \rightarrow \bar{B}^{0}} & =\frac{1}{2 \tau_{B}} e^{-t / \tau_{B}}\left(1-\cos \Delta m_{d} t\right) .
\end{aligned}
$$

Measurements of the frequencies of $B^{0}$ and $B_{S}^{0}$ oscillations can potentially constrain the magnitudes of the CKM matrix elements $V_{t d}$ and $V_{t s}$, where in the ratio of $\Delta m_{d} / \Delta m_{s}$ several theoretical uncertainties cancel out

$$
\frac{\Delta m_{d}}{\Delta m_{s}}=\frac{m_{B^{0}}}{m_{B_{S}^{0}}} \frac{\eta_{B^{0}}^{\mathrm{QCD}}}{\eta_{B_{S}^{0}}^{\mathrm{QCD}}} \frac{f_{B^{0}}^{2} B_{B^{0}}}{f_{B_{S}^{0}}^{2} B_{B_{S}^{0}}} \frac{\left|V_{t d}\right|^{2}}{\left|V_{t s}\right|^{2}} .
$$

Here, $f_{B}$ is the weak $B$ decay constant, $B_{B}$ the bag parameter of the $B$ meson, and $\eta^{\mathrm{QCD}}$ are QCD corrections which are in the order of one.

In general, a time dependent mixing measurement requires the knowledge of the flavour of the $B$ meson at production and at decay, as well as the proper decay time of the $B$ meson. Experimentally, the flavour of the $B$ meson is determined at the time of its decay from the observed decay products like the charge of the lepton from a semileptonic $B$ decay. The flavour at production time can be determined in various ways, employing either the second $b$-flavoured hadron in the event, or the charge correlation with particles produced in association with the $B$ meson. We report about two recent time dependent $B \bar{B}$ mixing results from CDF, which exploit these two ways of tagging the $B$ flavour at the origin.

\subsection{1 $B \bar{B}$ Mixing in $e \mu$ Events}

For this analysis the $e \mu$ trigger data are used, where both leptons are assumed to come from the semileptonic decay of both $b$ hadrons in the event: $b_{1} \rightarrow e X$ and $b_{2} \rightarrow \mu X$. This means, the flavour of the $B$ meson at decay is tagged by 
its semileptonic decay, while the semileptonic decay of the other $b$ hadron in the event tags the $B$ flavour at production. The requirement $m_{e \mu}>5 \mathrm{GeV} / c^{2}$ ensures that both leptons originate from two $b$ hadrons and not from a sequential decay of one $b$ hadron: $b \rightarrow c \ell_{1} X$, with $c \rightarrow \ell_{2} X$.

The principle of this analysis is to search for an inclusive secondary vertex associated with one of the leptons. The decay length of this vertex and the momenta of the tracks associated with the lepton provide an estimate of the $c \tau$ of the $B$ meson. The boost resolution for this technique is about $21 \%$ for electrons and about $24 \%$ for muons. In order to search for an inclusive secondary vertex, a modified version of the SVX $b$ tagging alogrithm, which was successfully used in the search for $b$ tags in top quark events (see Sec. 2.5), has been used. This algorithm has been tuned for high efficiency near $c \tau=0$, with the efficiency reaching a plateau of about $40 \%$ for $c \tau>500 \mu \mathrm{m}$ according to a $\mathrm{MC}$ study.

The important task of this analysis is to determine the sample composition, the fraction of events which come from $b \bar{b}$ decays with respect to events from $c \bar{c}$ or background events with at least one fake lepton. We find to a good approximation that the fake electron events are a subset of the fake muon events due to the higher $p_{\mathrm{t}}$ cut. Other backgrounds arise from sequential $b \rightarrow c \rightarrow \ell$ decays. The sample composition has been estimated from several kinematic quantities, like the $p_{\mathrm{t}}^{\text {rel }}$ distribution or the invariant mass of the tagged secondary vertex. Here, $p_{\mathrm{t}}^{\text {rel }}$ is defined as the transverse momentum of the lepton with respect to the highest $p_{\mathrm{t}}$ track in a cone around the lepton. An example of the determination of the sample composition is shown in Fig. 20a). The fitted fractions from $b \bar{b}, c \bar{c}$, and fake events are displayed. The final sample composition is given in Table 8, which shows that more than $80 \%$ of the events originate from $b \bar{b}$ decays.

From a fit to the like-sign lepton fraction as a function of $c \tau$ the mixing frequency $\Delta m_{d}$ is extracted as shown in Fig. 20 b). The fit includes components for direct and sequential $b$ decays, $c \bar{c}$, and fake events. In about $16 \%$ of the events with a secondary vertex around one lepton, a secondary vertex is also found around the other lepton. These events enter the like-sign fraction distribution twice and we allow for a statistical correlation between the two entries. The final fit result is

$$
\Delta m_{d}=(0.45 \pm 0.05 \pm 0.05) \mathrm{ps}^{-1}
$$

where the dominant systematic error arises from the uncertainty in the sample composition. 
CDF PRELIMINARY
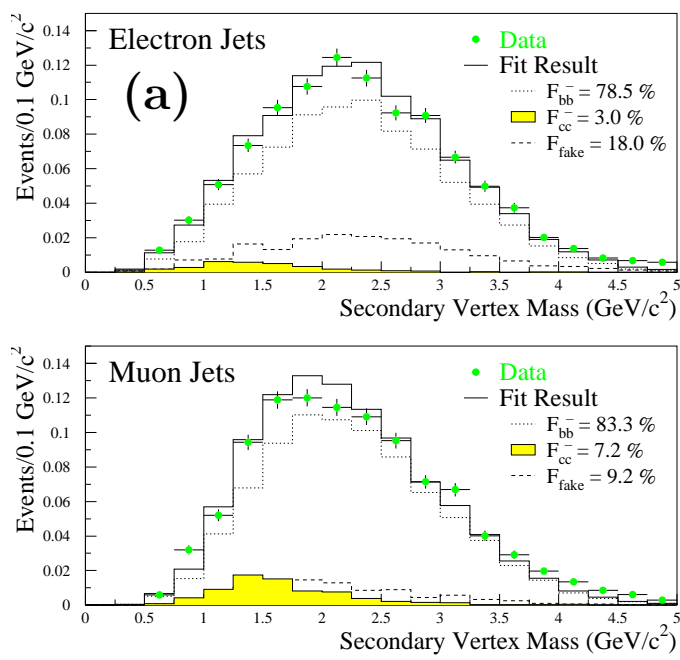

CDF PRELIMINARY

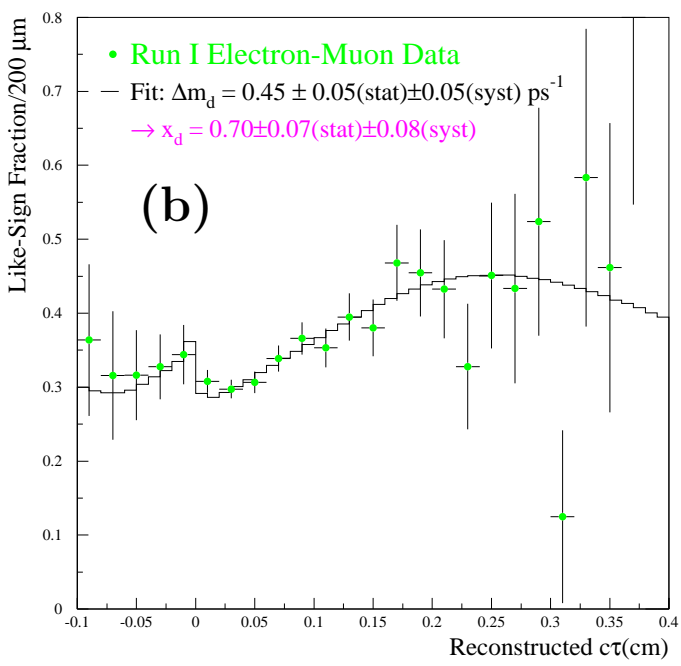

Figure 20: (a) Example of the determination of the sample composition in the $e \mu$ mixing analysis using the invariant mass of the secondary vertex. The fitted fractions from $b \bar{b}, c \bar{c}$, and fake events are shown. The terms 'e Jet' and ' $\mu$ Jet' indicate that the secondary vertex is associated with the electron or muon. In (b) the fit to the like-sign fraction versus $c \tau$ is displayed.

\begin{tabular}{|l|c|c|}
\hline Component & $e$ Jet & $\mu$ Jet \\
\hline \hline Fake e with Real $\mu$ & $\leq 1 \%$ & $\leq 1 \%$ \\
Fake $\mu$ Fraction & $(15 \pm 4) \%$ & $(7 \pm 3) \%$ \\
\hline$c \bar{c}$ events & $(2 \pm 2) \%$ & $(4 \pm 3) \%$ \\
\hline$b \bar{b}$ events & $(83 \pm 5) \%$ & $(89 \pm 4) \%$ \\
\hline Sequential $e$ & $(8.8 \pm 1.3) \%$ & $(7.9 \pm 1.2) \%$ \\
Sequential $\mu$ & $(13.6 \pm 2.0) \%$ & $(16.5 \pm 2.5) \%$ \\
\hline
\end{tabular}

Table 8: Final sample composition of the $e \mu$ mixing analysis. The terms ' $e$ Jet' and ' $\mu$ Jet' indicate that the secondary vertex is associated with the electron or muon. The sequential fractions are part of the $b \bar{b}$ component. 

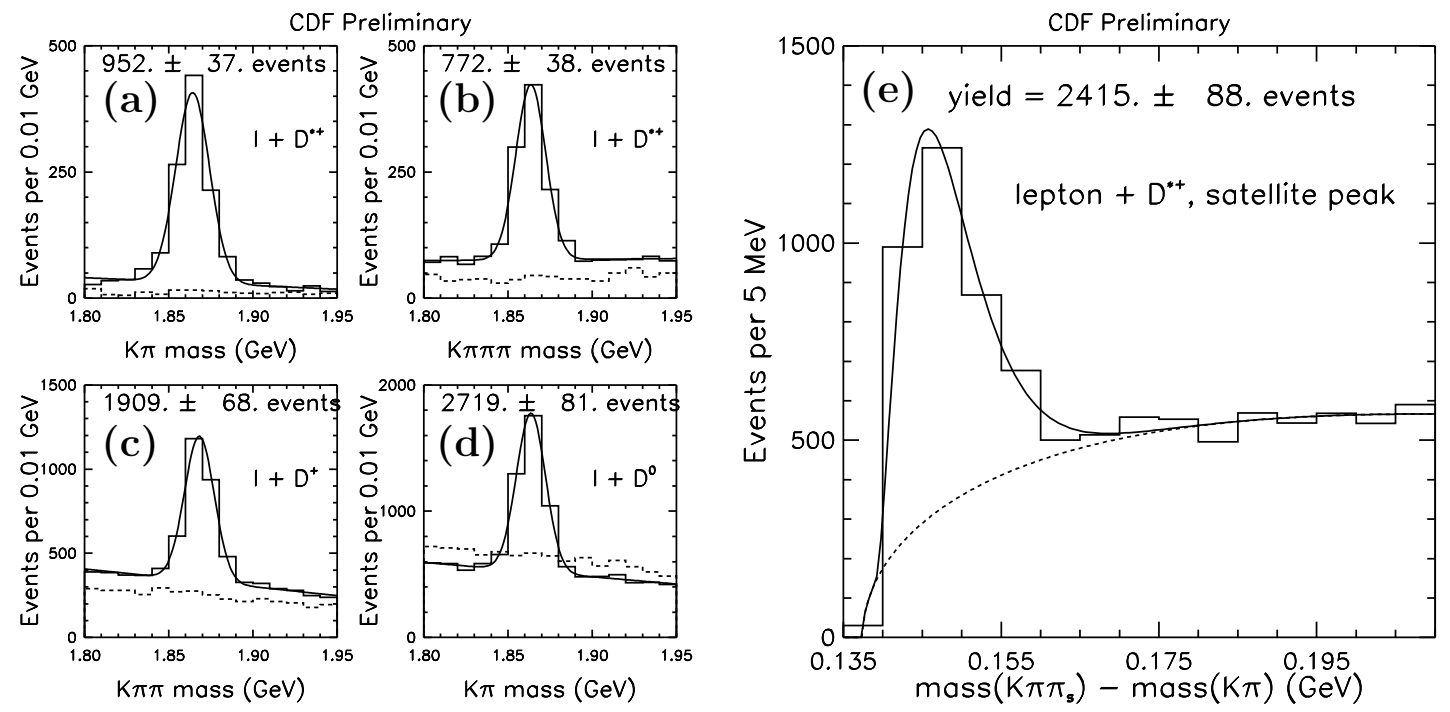

Figure 21: Invariant mass distribution of $D^{(*)}$ candidaites used in the $B$ mixing analysis using partially reconstructed $B \rightarrow D^{(*)} \ell X$ decays: (a) $\ell D^{*+}$ with $D^{*+} \rightarrow$ $D^{0} \pi^{+}, D^{0} \rightarrow K^{-} \pi^{+}$, (b) $\ell D^{*+}$ with $D^{*+} \rightarrow D^{0} \pi^{+}, D^{0} \rightarrow K^{-} \pi^{+} \pi^{+} \pi^{-}$, (c) $\ell D^{+}$ with $D^{+} \rightarrow K^{-} \pi^{+} \pi^{+}$, (d) $\ell D^{0}$ with $\ell D^{0} \rightarrow K^{-} \pi^{+}$, where the $D^{0}$ is not from a $D^{*+}$, and (e) $\ell D^{*+}$ with $D^{*+} \rightarrow D^{0} \pi^{+}, D^{0} \rightarrow K^{-} \pi^{+} \pi^{0}$, where the $\pi^{0}$ is not reconstructed.

\subsection{2 $B \bar{B}$ Mixing in $\ell D^{(*)}$ Events}

For this analysis $B$ mesons are reconstructed through their semileptonic decays $B \rightarrow D^{(*)} \ell X$ (see also Sec. 3.1.2). The analysis starts with the single lepton trigger data and reconstructs $D^{(*)}$ meson candidates in a cone around the trigger electron or muon in the following channels:

$$
\begin{array}{lll}
\bar{B}^{0} \rightarrow D^{*+} \ell^{-} \nu, \quad D^{*+} \rightarrow D^{0} \pi^{+}, & D^{0} \rightarrow K^{-} \pi^{+}, \\
& & D^{0} \rightarrow K^{-} \pi^{+} \pi^{+} \pi^{-}, \\
& D^{0} \rightarrow K^{-} \pi^{+} \pi^{0},\left(\pi^{0}\right. \text { not reconstructed) } \\
\bar{B}^{0} \rightarrow D^{+} \ell^{-} \nu, \quad D^{+} \rightarrow K^{-} \pi^{+} \pi^{+}, & \\
B^{-} \rightarrow D^{0} \ell^{-} \nu, \quad D^{0} \rightarrow K^{-} \pi^{+} & \text {(veto } D^{*+} \text { candidates). }
\end{array}
$$

Tracks with impact parameters significantly displaced from the primary vertex are selected in order to decrease combinatorial backgrounds. The signals are identified as peaks in the invariant mass spectra of the charm decay products as shown in Fig. 21 for the different $D^{(*)}$ decay modes.

The $D^{(*)}$ candidates are intersected with the lepton to find the $B$ decay vertex. 
Since the $B$ meson is not fully reconstructed, its $c \tau_{B}$ cannot be directly obtained. The boost of the $B$ meson is determined from the observed decay products and a $\beta \gamma$ correction is applied as obtained from a Monte Carlo simulation.

In order to tag the $B$ flavour at production, we use a 'same side tagging' (SST) algorithm, which exploits the correlation between the $B$ flavour and the charge of tracks from either the fragmentation process or $B^{* *}$ resonances. 34 In this analysis no attempt is made to differentiate the sources of correlated pions. To study the correlation between the flavour of the $B$ meson and the charged particles produced in association with it, we consider all tracks that are within an $\eta$ - $\phi$ cone of radius 0.7 centered around the direction of the $B$ candidate. Since the $B$ meson is only partially reconstructed, we approximate this direction with the momentum sum of the lepton and charm hadron.

The tracks considered as tags should be consistent with the hypothesis that they originate from the fragmentation chain or the decay of $B^{* *}$ mesons, i.e. that they originate from the primary vertex of the event. All tracks with transverse momentum $p_{\mathrm{t}}>0.4 \mathrm{GeV} / c$ are therefore required to satisfy $d_{0} / \sigma_{d_{0}}<3$, where $d_{0}$ is the distance of closest approach of the track trajectory to the estimated $B$ production position, and $\sigma_{d_{0}}$ is the estimated error on this quantity.

String fragmentation models indicate that the velocity of the fragmentation particles, that we seek for our tag, is close to the velocity of the $B$ meson. Similarly, pions from $B^{* *}$ decays should also have a velocity that is close to the velocity of the $B$ meson. In particular, the relative-transverse momentum $\left(p_{\mathrm{t}}^{\mathrm{rel}}\right)$ of the particle with respect to the combined momentum of the $B$ momentum plus particle momentum, should be small. Of the candidate tracks, we select as the tag the track that has the minimum component of momentum $p_{\mathrm{t}}^{\text {rel }}$ orthogonal to the momentum sum of that track, the lepton, and the $D$ meson. The efficiency for finding such a tag is about $72 \%$ for this algorithm.

Since we know the flavour of the $B$ meson at decay from the $D^{(*)} \ell$ signature, we compare the number of right-sign $\left(N_{R S}\right)$ correlations to the number of wrong$\operatorname{sign}\left(N_{W S}\right)$ tags as a function of $c \tau$. For the $B^{0}$ meson we expect the asymmetry $A(t)$ to be:

$$
A(t)=\frac{N_{R S}(t)-N_{W S}(t)}{N_{R S}(t)+N_{W S}(t)}=D \cdot \cos \left(\Delta m_{d} t\right),
$$

where $D$ is the dilution of the same side tagging algorithm. $D$ is also often expressed in terms of the mistag fraction $w$ as $D=1-2 w$. In our analysis we fit 

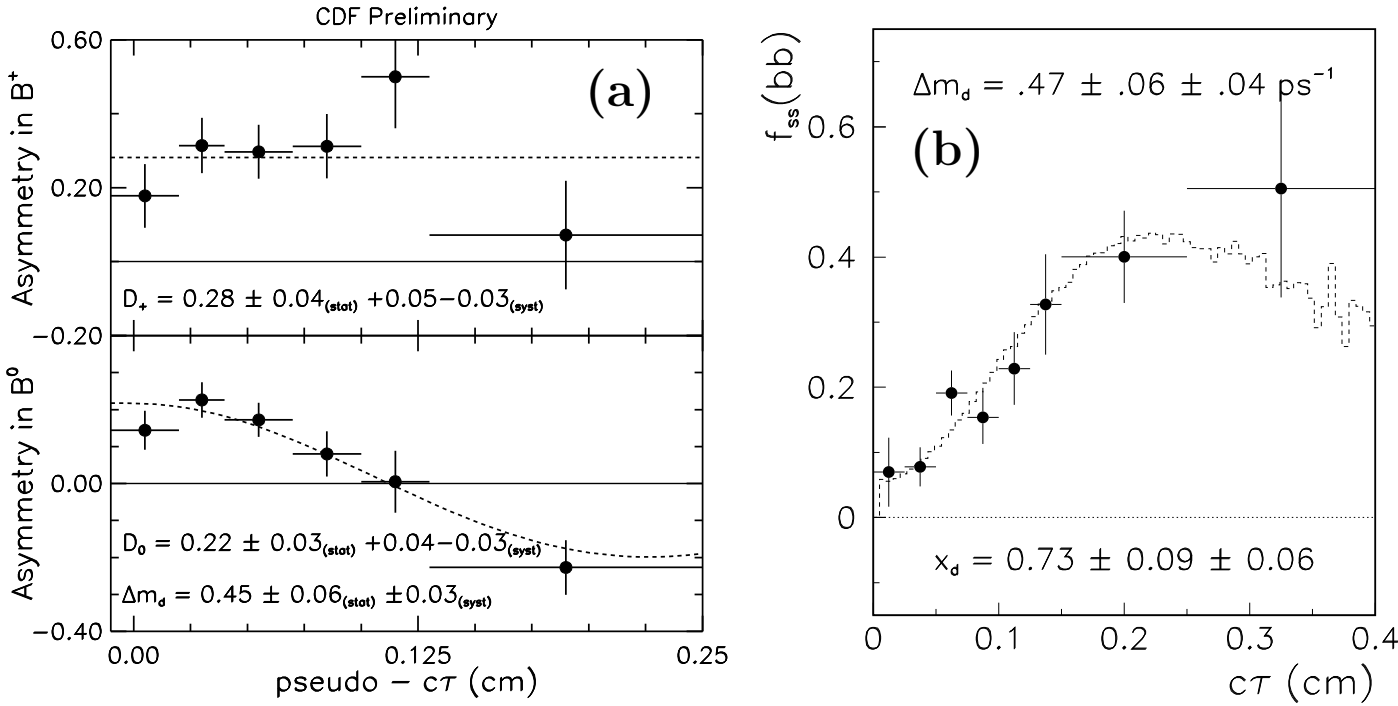

Figure 22: (a) Time dependent asymmetry for the $D^{(*)} \ell$ mixing analysis, where the top plot shows the measured asymmetry for $B^{+}$while the bottom plot represents the asymmetry for $B^{0}$. (b) Fitted like-sign fraction versus $c \tau$ for a $B$ mixing analysis using a jet charge and soft lepton tag.

for both $\Delta m_{d}$ and $D$.

To obtain the asymmetry for $B^{0}$ and $B^{+}$mesons, we correct for the fact that each $D^{(*)} \ell$ signal has contributions from both neutral and charged $B$ mesons via $D^{* *}$ decays. We correct for this cross talk by performing a fit bin by bin in $c \tau$. The inputs to the fit are the raw asymmetries as measured in each sample for a given $c \tau$ bin, and parameters describing the $D^{* *}$ composition in semileptonic $B$ decays. We fit the corrected $B^{0}$ asymmetry as a function of $c \tau$ to a cosine convoluted with the $c \tau$ resolution function, and extract the mixing frequency $\Delta m_{d}$ as well as the dilution $D$ of the same side tagging algorithm as shown in Fig. 22a). We also determine the asymmetry for the charged $B$ meson, which is flat in $c \tau$ as expected. We measure

$$
\Delta m_{d}=(0.45 \pm 0.06 \pm 0.03) p^{-1}
$$

and find $D=0.22 \pm 0.04_{-0.03}^{+0.04}$. We also determine the effective tagging efficiency to be $\varepsilon D^{2}=\left(3.4 \pm 1.0_{-0.9}^{+1.2}\right) \%$. The dominant systematic error arises from the uncertainty in the fraction of $D^{* *}$ in semileptonic $B$ decays.

In summary, we have reported two measurements of $\Delta m_{d}$ at CDF using $D^{(*)} \ell$ events with a same side tag as well as $e \mu$ dilepton events. There are more time 
dependent $B \bar{B}$ oscillation measurements in preparation at CDF, like a mixing analysis using a soft lepton tag (see Sec. 2.5) and a jet charge tag as shown in Fig. 22b). The result from this analysis is

$$
\Delta m_{d}=(0.47 \pm 0.06 \pm 0.04) p \mathrm{~s}^{-1}
$$

Since there is an event overlap of about $10 \%$ between this analysis and the $e \mu$ analysis, a combined CDF average has not yet been determined, but CDF's mixing

results start to become competitive with the LEP measurements. 35

\section{A Brief Look to the Future}

In Run I the luminosity of the Tevatron was limited by the antiproton current. The Fermilab accelerator complex is undergoing an upgrade to produce an order of magnitude higher luminosities in the Tevatron. The largest change will be to replace the Main Ring with the new Main Injector, which will be housed in a new tunnel. The Main Injector will provide higher proton intensity onto the antiproton production target, and larger aperture for the antiproton transfer into the Tevatron. After the completion and comissioning of the Main Injector, the Tevatron is scheduled to deliver luminosity again in summer of 1999. The centreof-mass energy will then be at $2.0 \mathrm{TeV}$. Luminosities of $2.0 \cdot 10^{32} \mathrm{~cm}^{-2} \mathrm{~s}^{-1}$ will be reached yielding an integrated luminosity of $2 \mathrm{fb}^{-1}$ delivered to the collider experiments within two years. The physics projections for Run II presented here assume $2 \mathrm{fb}^{-1}$ of integrated luminosity. In addition to the Tevatron upgrade the CDF and DØ experiments will undergo major detector upgrades, which are described in detail elsewhere.36.37

\subsection{Top Physics in Run II}

In Run II both collider detectors will be equipped with new and improved silicon vertex detectors which will for example enhance the efficiency to detect top decays. In addition, the Tevatron's centre-of-mass energy will be at $2.0 \mathrm{TeV}$, which will increase the top quark production cross section by $40 \%$. The estimated yield per experiment will be about 160 dilepton events, 990 lepton plus 4-jet events with one or more $b$ tags and for the top mass measurement about 240 lepton plus 4 -jet events where both $b$ jets are tagged. This will result in a statistical uncertainty 
on the top quark mass measurement of $1-2 \mathrm{GeV} / c^{2}$. The top production cross section will be known with a precision of better than $10 \%$. In addition, single top production can be studied, top polarization measurements can be performed, and rare top decays will be searched for. A more detailed overview of top quark physics in Run II can be found in Ref. 38

\subsection{B Physics in Run II}

In this section we concentrate on reviewing the future of $B$ physics at CDF by summarizing the prospects of measuring $C P$ violation in Run II. The $B$ physics goal is to measure the $C P$ asymmetry in $B^{0} \rightarrow J / \psi K_{S}^{0}$ and $B^{0} \rightarrow \pi^{+} \pi^{-}$determining $\sin 2 \beta$ and $\sin 2 \alpha$, respectively. $\mathrm{CDF}$ plans to also look for $C P$ violation in $B_{S} \rightarrow D_{S} K$ and $B \rightarrow D K$ probing $\sin 2 \gamma$.

$\mathrm{CDF}$ has the advantage of being an existing experiment that took plenty of data in Run I. We can use these data to study the ingredients for a future $C P$ violation measurement eg. in $B^{0} \rightarrow J / \psi K_{S}^{0}$. One input is the knowledge of the expected number of $J / \psi K_{S}^{0}$ events, which can be extrapolated from the $J / \psi K_{S}^{0}$ yield in CDF's current data. The second ingredient is the knowledge of the $B$ flavour at production. For this purpose CDF studies several $B$ flavour tagging methods at a hadron collider environment. The figure of merit to compare flavour tagging algorithms is the effective tagging efficiency $\varepsilon D^{2}$, where $\varepsilon$ is the efficiency of how often a flavour tag is applicable and $D$ is the dilution $D=\left(N_{R S}-N_{W S}\right) /\left(N_{R S}+N_{W S}\right)=1-2 w$. Here, $N_{R S}$ and $N_{W S}$ are the numbers of right and wrong sign tags, while $w$ is the mistag fraction (see also Sec. 3.2.2).

Figure 23 shows the invariant $J / \psi K_{S}^{0}$ mass distribution from CDF's current data corresponding to about $110 \mathrm{pb}^{-1}$. About 240 signal events with a signalto-noise ratio better than 1:1 are observed. This is currently the world's largest sample of $B^{0} \rightarrow J / \psi K_{S}^{0}$ and serves as proof that $B^{0} \rightarrow J / \psi K_{S}^{0}$ decays can be fully reconstructed in a hadron collider environment in a well understood way.

\subsection{1 $B$ Flavour Tagging Studies in Run I}

Several $B$ flavour tagging methods are studied with CDF data. One is referred to as 'same side tagging' (SST) which is described in more detail in Sec. 3.2.2. This method exploits charge correlations between $B$ mesons and charged particles produced in the fragmentation of the $b$ quarks. Such correlations are expected to 


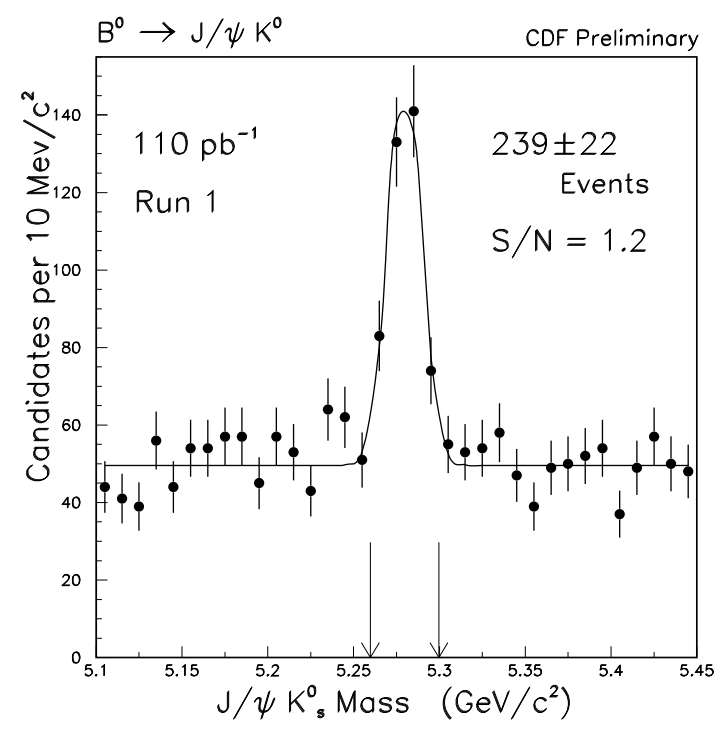

Figure 23: Run I signal of $B^{0} \rightarrow J / \psi K_{S}^{0}$ at CDF.

arise from particles produced in the fragmentation chain and from decays of the $L=1 B$ mesons (the $B^{* *}$ mesons). 4 Another way of tagging the flavour of a $B$ meson at production is to exploit the flavour of the other $B$ meson in the event. This can be done through a $B$ semileptonic decay (lepton tagging) or by counting the charge of the other $b$ jet (jet charge tagging). CDF has preliminary results on the effective tagging efficiency of these three methods.

Using a high statistics sample of partially reconstructed $B$ mesons from $\ell D^{0}$ $\left(B^{+}\right.$signature) and $\ell D^{+} / \ell D^{*+}\left(B^{0}\right.$ signature $)$ combinations, charge correlations between the $B$ candidate and tracks in its vicinity are studied as detailed in Sec. 3.2.2. In the case of charged $B$ mesons we measure $\varepsilon D^{2}=\left(5.7 \pm 1.5_{-1.2}^{+2.0}\right) \%$ $\left(D=\left(28 \pm 4_{-3}^{+5}\right) \%\right)$ for the same side tagging algorithm. For the neutral $B$ meson we obtain $\varepsilon D^{2}=\left(3.4 \pm 1.0_{-0.9}^{+1.2}\right) \%$ with $D=\left(22 \pm 3_{-3}^{+4}\right) \%$. The proof that this tagging method actually works is shown in Fig. 22a), where the asymmetry of right minus wrong sign tags normalized to the sum of both is plotted versus the $c \tau$ of the $B$ meson. The top plot is for $B^{+}$candidates and shows a flat behavior as expected. The bottom plots shows the same distribution for $B^{0}$ mesons, and exhibits an oscillatory behaviour as expected from $B \bar{B}$ mixing. This means applying same side tagging to a sample of partially reconstructed $B$ mesons results in a measurement of time dependent $B^{0}$ mixing. We also studied same side tagging with a sample of fully reconstructed $B \rightarrow J / \psi K^{(*)}$ decays. The results of $\varepsilon D^{2}=(4.0 \pm 1.9) \%(D=(33 \pm 8) \%)$ and $\varepsilon D^{2}=(1.5 \pm 3.0) \%(D=(19 \pm 19) \%)$ 
for $B^{+}$and $B^{0}$ mesons, respectively, are somewhat limited by statistics.

CDF also studied opposite side lepton tagging and obtains an effective tagging efficiency of $\varepsilon D^{2}=(0.6 \pm 0.1) \%$ for soft muon tagging and $\varepsilon D^{2}=(0.3 \pm 0.1) \%$ for soft electron tagging. The tagging algorithms are similar to the ones used in the top quark search as described in Sec. 2.5. The preliminary result for the effective tagging efficieny of jet charge tagging is $(1.0 \pm 0.3) \%$, which makes use of information from the silicon vertex detector. The proof that jet charge and soft lepton tagging actually works is shown in Fig. 22 $\mathrm{b}$ ), where the time dependent $B$

mixing measurement using these two tags is displayed. Combining the existing measurements on $B$ flavour tagging from Run I results in an effective tagging efficiency of $\approx 3.4 \%$.

\subsection{2 $C P$ Asymmetry in $B^{0} \rightarrow J / \psi K_{S}^{0}: \sin 2 \beta$}

For the measurement of $\sin 2 \beta$ in $B^{0} \rightarrow J / \psi K_{S}^{0}$ CDF expects about 15,000 $J / \psi K_{S}^{0}$ events. This event number will be obtained with a lower muon trigger threshold of $p_{\mathrm{t}}>1.5 \mathrm{GeV} / c$ compared to about $2.0 \mathrm{GeV} / c$ in Run I, improved muon coverage, and by also triggering on $J / \psi \rightarrow e^{+} e^{-}$. The effective tagging efficiencies are expected to improve with the upgraded detector. We expect $\varepsilon D^{2} \approx 2 \%$ for lepton tagging due to a better coverage for the lepton identification. For same side tagging we expect $\varepsilon D^{2} \approx 2 \%$ from a cleaner selection of fragmentation tracks with SVX II. Finally, for jet charge tagging we expect $\varepsilon D^{2} \approx 3 \%$ from an improved purity of the algorithm with 3-dimensional vertexing and the extented coverage of SVX II. Considering the overlap of all three tags by combining them, we expect a total $\varepsilon D^{2}$ of about $5.5 \%$ resulting in an uncertainty on $\sin 2 \beta$ of $\Delta \sin 2 \beta=0.09$.

\subsection{3 $C P$ Asymmetry in $B^{0} \rightarrow \pi^{+} \pi^{-}: \sin 2 \alpha$}

The key to measure the $C P$ asymmetry in $B^{0} \rightarrow \pi^{+} \pi^{-}$is to trigger on this decay mode in hadronic collisions. CDF plans to do this with a three level trigger system. On Level 1 two oppositely charged tracks with $p_{\mathrm{t}}>2 \mathrm{GeV} / c$ found with a fast track processor yield an accept rate of about $16 \mathrm{kHz}$. This will be reduced to about $20 \mathrm{~Hz}$ on Level 2 using impact parameter information $(d>100 \mu \mathrm{m})$. On Level 3 the full event information is available further reducing the trigger rate to about $1 \mathrm{~Hz}$. With this trigger we expect about $10,000 B^{0} \rightarrow \pi^{+} \pi^{-}$events in $2 \mathrm{fb}^{-1}$. Assuming the same effective tagging efficiency of $5.5 \%$ we expect an uncertainty 
on $\sin 2 \alpha$ of $\Delta \sin 2 \alpha=0.10$. Backgrounds from $B \rightarrow K \pi$ and $B \rightarrow K K$ decays can be extracted from the untagged signal by making use of the invariant mass distribution as well as CDF's $\mathrm{d} E / \mathrm{d} x$ capability in the central tracking chamber.

\subsubsection{CP Asymmetry in $B_{S} \rightarrow D_{S} K: \sin 2 \gamma$}

The $C P$ asymmetry in $\sin 2 \gamma$ completes the test of the unitarity triangle. The angle $\gamma$ can be probed via the decay $B_{S}^{0} \rightarrow D_{S}^{-} K^{+}$and $D_{S}^{+} K^{-}$, where both the mixed and the unmixed amplitudes can decay to the same final $D_{S}^{+} K^{-}$state. The interference between both amplitudes results in the weak phase $\gamma$, but also in a relative QCD phase which is expected to be small but a priori unknown. This measurement requires an all hadronic trigger with similar requirements as for $B^{0} \rightarrow \pi^{+} \pi^{-}$. We expect an overall efficiency times acceptance of $\approx 3 \cdot 10^{-4}$. Because of rapid $B_{S}^{0}$ oscillations a time dependent analysis is required but only a small sample of tagged events is expected at CDF in Run II.

The $C P$ asymmetry in $\sin 2 \gamma$ can also be explored via $B \rightarrow D K$. In this case the decays are self tagging and a time integrated analysis can be performed, but the theoretical uncertainties are large. Thus, performing a measurement of $\sin 2 \gamma$ through both modes will be a challenge at CDF in Run II.

\section{Conclusion}

In this article we have reviewed recent heavy flavour physics results from the Tevatron $p \bar{p}$ collider at Fermilab. We summarized the status of top quark physics at $\mathrm{CDF}$ and $\mathrm{D} \varnothing$. The top production cross section has been measured to be

$$
\sigma_{t \bar{t}}=\left(6.4_{-1.2}^{+1.3}\right) p \mathrm{~b}
$$

and the top quark mass is know with a precision of

$$
m_{\text {top }}=(175.0 \pm 3.9 \pm 4.5) \mathrm{GeV} / c^{2} .
$$

We also discussed recent $B$ physics results from the CDF collaboration. We summarized CDF's $B$ hadron lifetime measurements, which are very competitive with the LEP and SLC results, and discussed latest time dependent $B \bar{B}$ mixing results from CDF.

We also reviewed future prospects of top and $B$ physics at the Tevatron. In Run II, starting in 1999, both experiments will measure the top quark mass with 
a statistical uncertainty of $1-2 \mathrm{GeV} / \mathrm{c}^{2}$. The top production cross section will be known with a precision of better than $10 \%$. The future prospects of $B$ physics at $\mathrm{CDF}$ will concentrate on the discovery of $C P$ violation in the $B$ system. CDF expects to measure $\sin 2 \beta$ in $B^{0} \rightarrow J / \psi K_{S}^{0}$ with a precision of $\Delta \sin 2 \beta=0.09$. CDF will also search for $C P$ violation in $B^{0} \rightarrow \pi^{+} \pi^{-}$and expects to measure $\sin 2 \alpha$ with a precision of $\Delta \sin 2 \alpha=0.10$. A measurement of $\sin 2 \gamma$ will be challenging in Run II.

\section{Acknowledgements}

It is a pleasure to thank all friends and colleagues from the CDF and DØ collaboration for their excellent work and their help in preparing this talk. Special thanks go to L. Galtieri, J. Lys, M. Shapiro, and B. Winer. A constant source of inspiration and support is my wife Ann, who I would like to thank for her continuous understanding about the life of a physicist.

I would like to thank the CDF and DØ technical support staff at all CDF and $\mathrm{D} \varnothing$ institutions for their hard work and dedication. I also thank the Fermilab Accelerator Division for their hard and successful work in commissioning the machine for this physics run. The CDF and DØ experiments are supported by the U.S. Department of Energy; the U.S. National Science Foundation; the Istituto Nazionale di Fisica Nucleare, Italy; the Ministry of Science, Culture, and Education of Japan; the Natural Sciences and Engineering Research Council of Canada; the National Science Council of the Republic of China; the Commissariat à L'Energie Atomique in France, the Ministry for Atomic Energy and the Ministry of Science and Technology Policy in Russia, CNPq in Brazil, the Departments of Atomic Energy and Science and Education in India, Colciencias in Colombia, CONA-CyT in Mexico, the Ministry of Education, Research Foundation and KOSEF in Korea, and the A. P. Sloan Foundation.

I gratefully acknowledge the organizers of this inspiring meeting which was a genuine pleasure to attend. I am sorry for the late submission of this manuscript but as Albert Einstein already pointed out, time is relative: "Put your hand on a hot stove for a minute, and it seems like an hour. Sit with a pretty girl for an hour, and it seems like a minute. THAT's relativity." 


\section{References}

[1] S.W. Herb et al., Phys.Rev.Lett. 39 (1977) 252.

[2] Ch. Berger et al. (PLUTO), Phys.Lett. B76 (1978) 243;

C.W. Darden et al. (DASP), Phys.Lett. B76 (1978) 246.

[3] S. Behrends et al. (CLEO), Phys.Rev.Lett. 50 (1983) 881.

[4] W. Behrends et al. (JADE), Phys.Lett. B146 (1984) 437.

[5] H. Albrecht et al. (ARGUS), Phys.Lett. B192 (1987) 245.

[6] Particle Data Group, Phys.Rev. D54 (1996) 1.

[7] D.P. Barber et al. (MARK-J), Phys.Lett. B85 (1979) 463, Phys.Rev.Lett. 44 (1980) 1722;

Ch. Berger et al. (PLUTO), Phys.Lett. B86 (1979) 413, Phys.Rev.Lett. 45 (1980) 1533;

W. Bartel et al. (JADE), Phys.Lett. B88 (1979) 171, B89 (1979) 136,

Phys.Lett. B99 (1981) 277;

R. Brandelik et al. (TASSO), Phys.Lett. B133 (1983) 499;

B. Adeva et al. (MARK-J), Phys.Rev.Lett. 50 (1983) 799, 51 (1983) 433,

Phys.Lett. B152 (1985) 439, Phys.Rev. D34 (1986) 681;

M. Althoff et al. (TASSO), Phys.Lett. B138 (1984) 441, Z.Phys. C22 (1984) 307;

H.J. Behrend et al. (CELLO), Phys.Lett. B144 (1984) 297.

[8] H. Yoshida et al. (VENUS), Phys.Lett. B198 (1987) 570;

H. Sagawa et al. (AMY), Phys.Rev.Lett. 60 (1988) 93;

I. Adachi et al. (TOPAZ), Phys.Rev.Lett. 60 (1988) 97;

S. Igarashi et al. (AMY), Phys.Rev.Lett. 60 (1988) 2359;

K. Abe et al. (VENUS), Phys.Lett. B234 (1990) 382.

[9] G.S. Abrams et al. (MARK II), Phys.Rev.Lett. 63 (1989) 2447.

[10] M.Z. Akrawy et al. (OPAL), Phys.Lett. B236 (1990) 364;

D. Decamp et al. (ALEPH), Phys.Lett. B236 (1990) 511;

P. Abreu et al. (DELPHI), Phys.Lett. B242 (1990) 536;

O. Adriani et al. (L3), Phys.Rep. 236 (1993) 1.

[11] G. Arnison et al. (UA1), Phys.Lett. B147 (1984) 493.

[12] C. Albajar et al. (UA1), Z.Phys. C37 (1988) 505. 
[13] T. Akesson et al. (UA2), Z.Phys. C46 (1990) 179.

[14] F. Abe et al. (CDF), Phys.Rev.Lett. 64 (1990) 174.

[15] F. Abe et al. (CDF), Phys.Rev.Lett. 68 (1992) 447, Phys.Rev. D45 (1992) 3921.

[16] S. Abachi et al. (DØ), Phys.Rev.Lett. 72 (1994) 2138.

[17] F. Abe et al. (CDF), Phys.Rev.Lett. 73 (1994) 225.

[18] F. Abe et al. (CDF), Phys.Rev. D50 (1994) 2966.

[19] F. Abe et al. (CDF), Phys.Rev.Lett. 74 (1995) 2626.

[20] S. Abachi et al. (DØ), Phys.Rev.Lett. 74 (1995) 2632.

[21] F. Abe et al. (CDF), Nucl.Instr.Methods A271 (1988) 387, and references therein.

[22] D. Amidei et al. (CDF), Nucl.Instr.Methods A350 (1994) 73.

[23] S. Abachi et al. (DØ), Nucl.Instr.Methods A338 (1994) 185, and references therein.

[24] E. Laenen, J. Smith, and W.L. Van Neerven, Nucl.Phys. B369 (1992) 543, Phys.Lett. B321 (1994) 254.

[25] P. Nason, S. Dawson, and R.K. Ellis, Nucl.Phys. B303 (1988) 607.

[26] E.L. Berger and H. Contopanagos, Phys.Rev. D54 (1996) 3085.

[27] S. Catani et al., Phys.Lett. B378 (1996) 329.

[28] N. Cabibbo, Phys.Rev.Lett. 10 (1963) 531;

M. Kobayashi, T. Maskawa, Prog.Theor.Phys. 49 (1973) 652.

[29] C. Albajar et al. (UA 1), Phys.Lett. B186 (1987) 247;

Phys.Lett. B256 (1991) 221; erratum: ibid, B262 (1991) 497.

[30] S. Abachi et al. (DØ), Phys.Rev.Lett. 74 (1995) 3548;

Phys.Lett. B370 (1996) 239.

[31] I.I. Bigi, Nuovo Cim. A109 (1996) 713.

[32] M. Neubert, Int.J.Mod.Phys. A11 (1996) 4173.

[33] J.D. Richman, Proceedings of the 28th International Conference on High Energy Physics, 25-31 July 1996, Warsaw, Poland.

[34] M. Gronau, A. Nippe and J. Rosner, Phys.Rev. D47 (1993) 1988. 
[35] L. Gibbons, Proceedings of the 28th International Conference on High Energy Physics, 25-31 July 1996, Warsaw, Poland.

[36] The CDF Collaboration, FERMILAB-Pub-96/390-E (1996).

[37] The DØ Collaboration, PAC-Report, Sept. 1996.

[38] Report from the TeV2000 Study Group, FERMILAB-Pub-96/082-E (1996). 